Research Article

\title{
Optimal Design and Acoustic Assessment of Low-Vibration Rotor Blades
}

\author{
G. Bernardini, ${ }^{1}$ E. Piccione, ${ }^{1}$ A. Anobile, ${ }^{2}$ J. Serafini, ${ }^{1}$ and M. Gennaretti ${ }^{1}$ \\ ${ }^{1}$ Department of Engineering, Roma Tre University, 00146 Rome, Italy \\ ${ }^{2}$ Department of Mechanical, Materials and Manufacturing Engineering, The University of Nottingham, Nottingham NG7 2RD, UK
}

Correspondence should be addressed to J. Serafini; serafini@uniroma3.it

Received 28 December 2015; Accepted 2 March 2016

Academic Editor: Jiun-Jih Miau

Copyright (C) 2016 G. Bernardini et al. This is an open access article distributed under the Creative Commons Attribution License, which permits unrestricted use, distribution, and reproduction in any medium, provided the original work is properly cited.

An optimal procedure for the design of rotor blade that generates low vibratory hub loads in nonaxial flow conditions is presented and applied to a helicopter rotor in forward flight, a condition where vibrations and noise become severe. Blade shape and structural properties are the design parameters to be identified within a binary genetic optimization algorithm under aeroelastic stability constraint. The process exploits an aeroelastic solver that is based on a nonlinear, beam-like model, suited for the analysis of arbitrary curved-elastic-axis blades, with the introduction of a surrogate wake inflow model for the analysis of sectional aerodynamic loads. Numerical results are presented to demonstrate the capability of the proposed approach to identify low vibratory hub loads rotor blades as well as to assess the robustness of solution at off-design operating conditions. Further, the aeroacoustic assessment of the rotor configurations determined is carried out in order to examine the impact of low-vibration blade design on the emitted noise field.

\section{Introduction}

Main rotors play a fundamental role in helicopter dynamics, providing both lifting force and thrust, but, as a by-product, they are sources of vibrations and noise, primarily due to the nonaxial flow condition of most of their typical mission profiles. The reduction of these annoying effects is of primary interest for rotors designers, since vibrations strongly affect fatigue life of structures, maintenance costs, onboard instrumentation efficiency, and passengers and pilot comfort as well as acoustic disturbance inside the cabin; similar problems concern wind turbines due to wind shear effect. At the same time, the external noise emission causes community disturbance, thus limiting the public acceptance of helicopters for operations nearby populated areas.

In the last years, several innovative blade shapes have been investigated by manufacturers and researchers, in order to alleviate vibrations and noise caused by main rotor. In particular, advanced geometries characterized by curved elastic axis (with tip sweep and anhedral angles included) have shown to be effective to this purpose (see, e.g., $[1,2]$ ).
Such blades reduce normal tip Mach number and consequently drag due to compressibility, alleviate the interactions occurring between the blades and the vortices of the rotor wake (and hence the blade-vortex interaction- (BVI-) noise), and may significantly affect the rotor aeroelastic behavior, introducing strong bending-torsion coupling.

The objective of this paper is the presentation and application of an optimal design procedure aimed at identifying rotor blades that generate reduced vibratory hub loads. It is a very challenging goal, in that it deals with an inherently multidisciplinary, multidimensional, constrained minimization problem, characterized by nonlinear, multimodal objective functions (i.e., functions with several local minima in the design domain). Because of this, genetic algorithms (GAs) seem to be an appropriate approach to achieving the optimal solution. Indeed, they are able to escape local minima and search for the global optimum even in very complex problems allowing, at the same time, the implementation of very efficient computational algorithms due to their intrinsically parallel nature. 
In the past literature on similar topics, several approaches based on deterministic optimization methods can be found [3-7], whereas only a limited number of more recent works address the use of nondeterministic methods such as GAs, for the same aim of [8-10]. For the sake of computational efficiency, in both optimization methodologies, often a surrogate model for the direct evaluation of the objective function is adopted [4, 8-10]. Extensive reviews of works dealing with rotorcraft optimization are given in [11-13].

In this work a binary-based GA developed by the authors is adopted, where blade tip sweep angle, blade tip anhedral angle, and the distribution of the mechanical/structural properties are considered as design variables to be identified under aeroelastic stability constraint. Recently, this binarybased GA has been successfully applied for the optimal design of aircraft cabin noise control and marine propeller blades $[14,15]$. The interested readers may find details on GA optimization procedures in $[16,17]$, while a thorough survey of multidisciplinary design optimization techniques is presented in [18].

In the optimal design process, a fundamental role is played by the simulation of the aeroelastic behavior of the rotor, since vibratory loads are a by-product of the interaction between the blades and the complex aerodynamic field in which they operate. In the past years, several authors have developed structural models for curved/swept tip blades, usually solved through the finite element method approach. One of the earliest models suited for swept tip blades has been presented and successfully applied to hingeless rotors in $[19,20]$. Among the others, a formulation for blades with varying sweep, droop, twist angles, and platform has been introduced in [21], while more recently a curvilinear-axis blade formulation has been applied to horizontal-axis wind turbines [22]. In the optimization procedure proposed here, the rotor aeroelastic tool consists of an enhanced version of that recently developed by the authors, presented and validated in $[23,24]$. It is based on a nonlinear, beamlike model spatially integrated through a Galerkin approach, suited for the analysis of blades having arbitrarily curved elastic axis (including geometrical discontinuities as sweep and anhedral tip angles). A good trade-off between accuracy and computational efficiency is achieved by the identification and application of a surrogate model of the wake inflow used for sectional aerodynamic loads prediction, relying on a boundary element approach for the analysis of unsteady potential flows [25].

The numerical investigation will assess the capability of the proposed optimal design approach to identify rotor blades generating reduced vibratory hub loads as well as the robustness of the solution at off-design operating conditions. An analysis of the influence of wake inflow model used in the aeroelastic tool on the optimization process will be also presented. Furthermore, observing that vibratory hub loads and noise emission are usually strictly related (alleviation of vibrations often corresponds to noise increase and vice versa), the acoustic performances of the identified optimal rotor will be examined. To this purpose, a boundary element approach based on Farassat's Formulation 1A [26] for the solution of Ffowcs Williams and Hawkings' equation [27] is applied.

\section{Materials and Methods}

In the following, an outline of the aeroacoustoelastic and optimization tools developed by the authors is presented.

2.1. Rotor Aeroelastic Solver. For the purposes of this work, the availability of an efficient and accurate computational tool for the aeroelastic analysis of rotor blades having arbitrary shape is of paramount importance. The one applied here has been developed by the authors as a combination of a suited structural dynamics model with the aerodynamic loads given by a quasi-steady sectional formulation corrected with inflow contribution, in order to take into account the effect of wake vortices.

The rotor blade structural dynamics is described through a beam-like model, obtained as an enhanced version of the formulation presented by some of the authors in [23]. It is valid for slender, homogeneous, and isotropic rotating blades with curved elastic axis and includes spanwise variation of mass and stiffness properties as well as variable builtin pretwist, precone, sweep, and anhedral angles. Nonlinear strain-displacement relations are considered with the application of a second-order approximation scheme, in order to take into account the moderate displacements usually experienced by rotor blades. A detailed description of this blade structural model is presented in Appendix.

The distributed aerodynamic loads are modeled by the quasi-steady approximation of the sectional Greenberg theory [28] (see Appendix). Three-dimensional unsteady effects deriving from the wake vorticity are taken into account through the influence of the wake inflow on the relative velocity at the rear aerodynamic center of the blade cross sections. The evaluation of the inflow is obtained by a boundary element method (BEM) for the solution of the boundary integral equation approach presented in [25], suited for the analysis of potential flows around rotors in arbitrary motion. Simple analytical wake inflow models might also be applied.

The aeroelastic integrodifferential model derived by coupling these structural dynamics and aerodynamic formulations has been spatially integrated through application of the Galerkin approach. In particular, as outlined in Appendix, a spectral description of the curvature components in the undeformed-axis frame is considered, in order to develop a solver with good convergence properties even in presence of sweep and anhedral tip angles (i.e., for elastic axis shapes with discontinuous first-order derivatives). The projections are applied to the bending and torsion moment equilibrium equations, with coinciding sets of trial and test functions (note that, in addition to shear undeformable assumption, the assumption of inextensible elastic axis has been adopted akin to the approach in [29]). Because of the test functions chosen, the present approach yields equations that are strongly related to those that could be derived from application of the Rayleigh-Ritz approach (see Appendix).

The aeroelastic response to steady flight conditions is evaluated by integrating the set of ordinary time-differential equations through a harmonic balance approach $[30,31]$. Aeroelastic stability about the equilibrium solution is instead 
examined by eigenanalysis of the (numerically) linearized system [31].

2.2. Rotor Aeroacoustics Solver. The aeroacoustic solver used to evaluate the noise radiated by rotor blades is based on the boundary integral Farassat's Formulation 1A [26] for the solution of the Ffowcs Williams and Hawkings equation [27] that represents a rearrangement of the mass and momentum conservation laws into an inhomogeneous wave equation. It is composed of three separate integral time-retarded contributions known as thickness, loading, and quadrupole noise, each related to a specific mechanism of noise generation. The thickness term depends on blade geometry and kinematics of the problem; the loading term is related to blade airloads; whereas the quadrupole source contribution accounts for the possible nonlinear effects taking place in the flow field. The quadrupole field term has been neglected since the blade velocity is far from the transonic/supersonic regimes (as it is in the rotor configurations examined here). Thickness and loading noise terms are solved by a simple zeroth-order formulation applied to the blade surface discretized into panels, with the integrand functions assumed uniform in each panel and equal to the values at the panel centroid.

The aeroacoustic formulation is based on the knowledge of the aerodynamic loads distributed over the blade surface. Here, they are obtained from the same aerodynamic tool applied for validating the vibratory performance of the blade identified in the optimal design process (see Section 3).

2.3. The Optimal Blade Design Process. The blade optimization procedure applied in this work is driven by a binary-based genetic algorithm developed by the authors $[14,15]$. Genetic algorithms are probabilistic programming techniques that mimic the natural evolution in finding the optimal solution of a given problem [16]. In this process, potential solutions are called individuals and the whole set of individuals is called population. Each individual is identified by a string (chromosome) of binary digits (genes) ordered in a given sequence. The optimization procedure starts from a completely random-generated population and, at each step of the evolution process, individuals are quantitatively evaluated in terms of the corresponding value of the objective function. The population size in genetic algorithms is a crucial issue to consider when dealing with specific optimization problems, as it can seriously affect their efficiency. Indeed, a very small population (i.e., composed of few individuals) may lead to an unsatisfactory coverage of the problem domain as well as to sampling errors [32], while a large population can lead to high computational time, due to the number of the objective functions to be evaluated. Here, following [17], an estimate of the population size based on the variance of the objective functions is used.

Constraints are included in the optimization process through a quadratic extended interior penalty-function approach [33], which enhances the breeding possibility of individuals potentially able to generate good offspring. In this sense, constraints are taken into account indirectly, turning the constrained optimization process into a sequence of unconstrained minimization procedures. To build a new generation, the best individuals are selected on the basis of a fitness measure evaluated from the objective function and constraints. For the present analysis, a tournament operator is used based on a random selection of four parents, which are compared one versus one in two pairs and the couple of "winners" are selected to be parents of two children with two independent crossover operations. A single random-point crossover operator is used.

Once the mate is performed, a binary uniform mutation operation is applied to avoid premature convergence to local optima. This operator alters one or more binary digits (genes) in the chromosome by flipping it with a given probability. The amount of chromosome variations during the evolutionary process is controlled through a user-defined mutation probability factor, which is decreased during the optimization to reduce the impact of random mutations as the solution converges to an optimum. In order to prevent possible negative aspects of the evolution process and hence drive the solutions to get better over time, at each step of the optimization process, the best individuals (a given, userdefined percentage of the population size) are selected to become part of an elite group which is unchanged in the next generation. This technique, in addition to avoiding the possibility to obtain worse generation during the process, enhances its convergence properties $[34,35]$. The optimization procedure is iterated until either the chromosomes similarity (bit-string affinity) achieves a user-defined value [36], or the maximum number of iterations is reached.

Here, this optimization process is applied to reduce the vibratory hub loads generated by a helicopter rotor in forward flight. Following past works $[5,37,38]$, the goal is pursued by tailoring the structural, inertial, and aerodynamic properties of the rotor blade. In particular, the following design variables are considered: bending and torsional stiffnesses, mass per unit length (assumed to be uniformly distributed spanwise), and sweep and anhedral angles (defined in the 15\% long blade tip region). Simple distributions of the structural design variables are considered in that the main objective of this work consists in the assessment of the effectiveness of the proposed optimization methodology to helicopter rotors design. Anyway, more complex variables distributions might be introduced at the cost of increasing the computational effort required. Given that $N$-bladed rotor transmits to the hub periodic forces and moments of fundamental frequency $\mathrm{N} / \mathrm{rev}$, the objective function to be minimized in the optimization is a linear combination of the scalar norm of the $\mathrm{N} /$ rev harmonics of hub forces and moments.

The tailoring of inertial, structural, and geometrical properties of the blades for low-vibration purposes may affect the aeroelastic stability of the rotor and, at the same time, the helicopter trim controls setting. Therefore, the optimal blade design process includes constraints regarding the equilibrium trim conditions and the rotor stability. Specifically, at each iteration, trim control settings are reevaluated together with the vibratory loads through an aeroelastic trim procedure, whereas the stability is imposed by setting a minimum acceptable value of the resulting critical damping. Finally, in order to have a dynamic behavior of the optimal blade similar to that of the reference one (in terms of eigenfrequencies 
TABLE 1: Blade design variables.

\begin{tabular}{lcccc}
\hline & Baseline & Single-point opt. (Drees inflow) & Single-point opt. (LIN surrogate) & Multipoint opt. (LIN surrogate) \\
\hline$E I_{\eta} / m_{0} \Omega^{2} R^{4}$ & 0.01060 & 0.01283 & 0.00857 & 0.00898 \\
$E I_{\zeta} / m_{0} \Omega^{2} R^{4}$ & 0.03010 & 0.03514 & 0.02948 & 0.02570 \\
$G J / m_{0} \Omega^{2} R^{4}$ & 0.00147 & 0.00104 & 0.00113 & 0.00188 \\
$m / m_{0}$ & 1.0 & 1.053 & 1.188 & 1.033 \\
$\Lambda_{A}[\mathrm{deg}]$ & 0.0 & 4.24 & -4.4 & -4.1 \\
$\Lambda_{S}[\mathrm{deg}]$ & 0.0 & 19.29 & 23.7 & 18.2 \\
\hline
\end{tabular}

and blade deflections amplitude), upper and lower bounds on the design variables are imposed. In particular, with respect to the baseline values, the largest acceptable variations of bending and torsional stiffnesses are selected to be equal to $30 \%$, and the largest acceptable variation of distributed mass is $20 \%$, while the tip sweep angle, $\Lambda_{S}$ (positive backwards), and the tip anhedral angle, $\Lambda_{A}$ (positive downwards), are constrained to be $-20^{\circ} \leq \Lambda_{S} \leq 30^{\circ}$ and $-10^{\circ} \leq \Lambda_{A} \leq 15^{\circ}$, respectively.

All the numerical results that are presented in the next section have been obtained by considering populations with individuals identified through a chromosome string of 24 digits (which allows a very fine resolution of the design variable range). The optimization process is iterated until a bit-string affinity of $85 \%$ or a maximum number of 50 populations is reached.

\section{Results and Discussion}

In this section, application strategies and effectiveness of the proposed optimal approach for the design of rotor blades generating low vibratory hub loads are examined. In particular, four main issues are investigated: (i) single-point and multipoint optimization algorithms performance, (ii) effect of aerodynamic modeling on the optimization process, (iii) robustness of optimal blade design in off-design flight conditions, and (iv) impact of low-vibration blade design on the emitted noise.

The optimal design processes have been applied to a Bo105 -like rotor with four hingeless blades and solidity $\sigma=0.07$, operating at Lock number $\gamma=5.5$ and thrust coefficient $C_{T}=$ 0.005 . The baseline values of the blade design variables are given in Table 1 .

Akin to the baseline rotor blade, the optimized blade has been assumed to have uniform structural properties. All computations have been carried out using nine shape functions in the modal description of each structural dof, whereas five harmonics have been included in the harmonic balance solution (these discretization parameters guarantee aeroelastic converged results).

The optimization process includes a constraint imposing aeroelastic stability in the design advancing flight condition (in order to cover the whole flight envelope more than one condition may be considered). However, noting that hover flight is usually critical in terms of aeroelastic stability, this constraint has been imposed in hovering as well. Control settings have been determined as those corresponding to the rotor momentum trim.
3.1. Single-Point Optimization. First, a single-point optimization algorithm has been applied, assuming the design flight condition at advance ratio $\mu=0.3$. The analytical Drees formula has been considered as the first candidate for wake inflow model in the aeroelastic tool, in that yielding an extremely computationally efficient solution process, fully suitable for use in GAs.

In this case, the optimization led to a reduction of about $84 \%$ of the objective function, $\widehat{J}$, given by the following linear combination of the scalar norm of the $4 / \mathrm{rev}$ harmonics of hub forces and moments:

$$
\widehat{J}=\left(F_{x}^{2}+F_{y}^{2}+F_{z}^{2}\right)^{1 / 2}+\left(M_{x}^{2}+M_{y}^{2}+M_{z}^{2}\right)^{1 / 2} .
$$

This is achieved with the identified optimal blade design variables given in Table 1 . With respect to the baseline values, the optimal design shows an increase of blade mass and bending stiffnesses, a reduction of torsional stiffness, a rearward tip sweep angle of $19.3 \mathrm{deg}$, and a downward tip anhedral angle of 4.2 deg. Figure 1 (a) depicts the $4 /$ rev vibratory hub loads from baseline and optimal rotors evaluated through the aeroelastic model used in the optimization procedure, demonstrating that very good reductions, ranging from $60 \%$ to $85 \%$, are achieved.

Then, the optimal blade design has been validated against application of an aerodynamic model more accurate than that used in the synthesis process. To this purpose, a highfidelity aeroelastic model based on the numerical free-wake inflow evaluated through the BEM formulation mentioned in Section 2.1 has been considered. This analysis confirms the aeroelastic stability of the optimal rotor configuration, but a reduction of the objective function with respect to the baseline configuration of only $5 \%$ is obtained. To better understand the results deterioration, the vibratory hub loads of both baseline and optimal rotor from the high-fidelity aeroelastic model are presented in Figure 1(b). From the comparison of Figures 1(a) and 1(b), it is apparent that the vibratory loads are very sensitive to the aerodynamic model used for their evaluation, with considerably higher values predicted by the high-fidelity aerodynamic model. Furthermore, the out-of-plane component of the hub forces, $F_{z}$, is significantly increased from its baseline value, whereas the in-plane force, $F_{x}$, and the torque moment, $M_{z}$, are weakly affected by the blade redesign. These results show that the sensitivity of the vibratory loads to the design variables as predicted by the two inflow models is very different (for some load components, even opposite), thus suggesting the need of using the more accurate aerodynamic model in rotor 


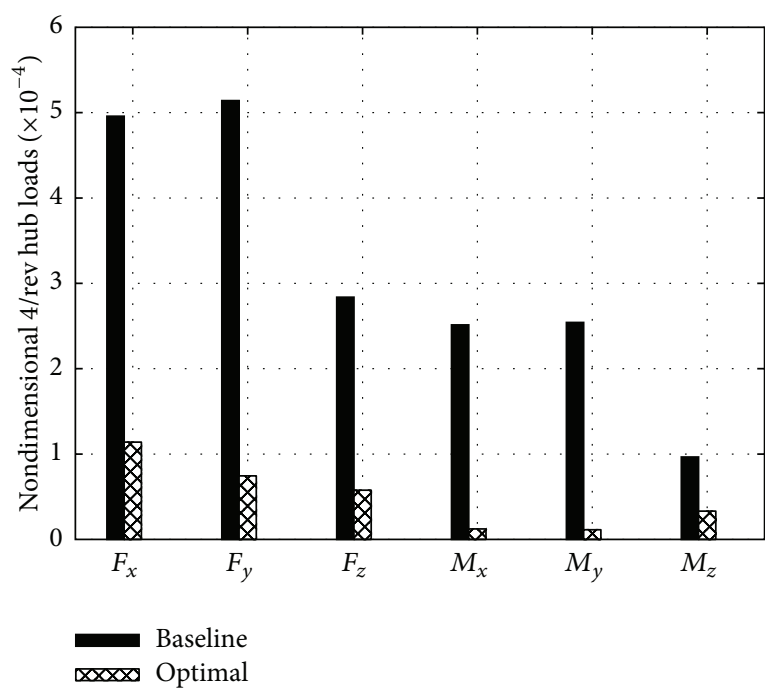

(a) Synthesis (Drees wake inflow)

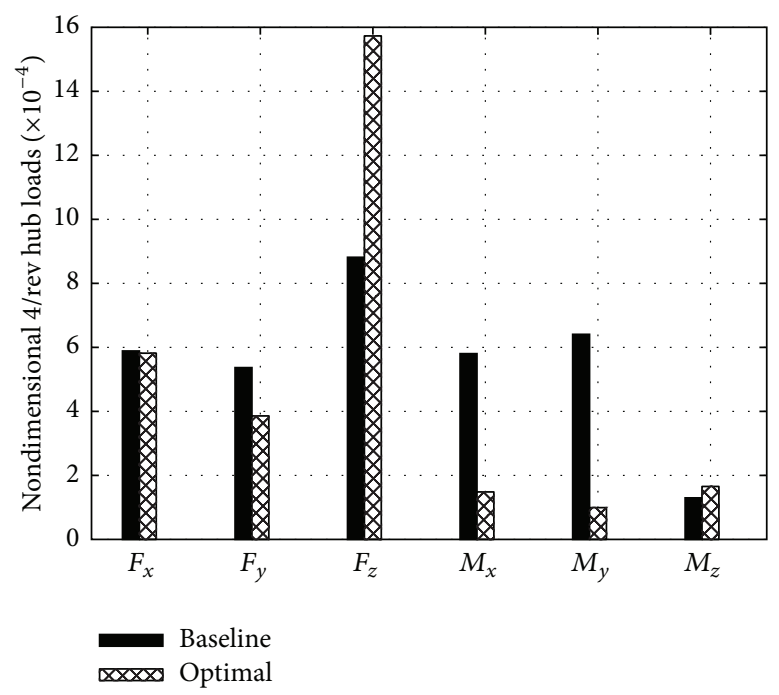

(b) Validation (BEM wake inflow)

FIGURE 1: Vibratory 4/rev hub loads: optimal versus baseline configuration at $\mu=0.3$.

aeroelastic optimization. Thus, the process has been repeated replacing the Drees model with the inflow numerically evaluated through the free-wake BEM in the aeroelastic tool. This allows the introduction of aerodynamic phenomena, like blade-vortex interactions, that may play an important role in the generation of vibratory loads and that are completely neglected in using simple analytical wake inflow models. However, noting that a direct use of the BEM solver in the optimization process would significantly decrease its computational efficiency, a surrogate model of the BEM wake inflow has been synthesized and used in the optimizer. This model is based upon a linear interpolation of a database of the BEM wake inflow, previously evaluated for a limited number of blade operating conditions falling in the domain of definition of the optimization problem in terms of both design variables and flight conditions. In order to limit the number of rotor disk wake inflow computations to be performed to define the surrogate model (rapidly increasing with the number of considered parameters), and considering that only onepoint and two-point optimizations have been performed, for the purposes of this work, a different surrogate model has been synthesized for each considered flight condition. Furthermore, for the database definition, nine blades have been considered, differing only in the values of the sweep and anhedral blade tip angles. Indeed, a preliminary sensitivity analysis has shown that the wake inflow is weakly affected by mechanical/structural blade properties.

Using this linear (LIN) surrogate inflow model in the optimization process, the optimal blade variables given in column 3 of Table 1 have been identified, showing decreased blade mass and bending stiffnesses with respect to the baseline values and an upward tip anhedral angle. This blade model yields a reduction of the objective function of $74 \%$ in the synthesis phase that is slightly lower than that obtained with the Drees inflow model. However, in this case, validating the optimal design against application of the high-fidelity aeroelastic solver based on the (nonsurrogate) BEM free-wake inflow model has given positive results. Indeed, the optimal blade has confirmed both a stable aeroelastic behavior and a significant (63\%) reduction of the examined objective function.

Figure 2(a) presents the comparison among vibratory hub loads given by (i) the baseline rotor, (ii) the optimal blade rotor within the optimal design process (synthesis), and (iii) the optimal blade rotor in the validation analysis. It demonstrates the effectiveness of the proposed design: the results obtained in the synthesis and validation phases are indeed quite similar, as only small discrepancies appear in the prediction of the in-plane force, $F_{y}$, of the out-of-plane force, $F_{z}$, and of the torque moment, $M_{z}$. The sensitivity of vibratory loads to variations of the design variables predicted by the surrogate wake inflow model is similar to that from the high-fidelity aerodynamic model, and hence it has proven to be well suited for rotor blade optimization applications. The validation of the optimal design against aerodynamic modeling variation has been performed also in terms of aeroelastic stability: the high-fidelity aeroelastic solver predicts a stable behavior of the optimal blade rotor both at $\mu=0.3$ and in hovering that are the two flight conditions considered for the stability constraint. Further, the 1/rev and 2/rev blade loads in the rotating frame, which do not contribute to the vibratory hub loads but stress blade root, are monitored in Figure 2(b). It shows that these loads, although not taken into account in the minimization process, at least in this case, are either practically unaffected or decreased, with the exception of the $2 /$ rev normal shear force that is subject to an increase of about $25 \%$. However, this can be considered as an acceptable minor drawback of the optimal design configuration.

Next, in order to assess the robustness of the design with respect to off-design flight conditions, vibratory hub loads and aeroelastic stability of the optimal rotor have been examined at advance ratio $\mu=0.15$. Akin to the case with 


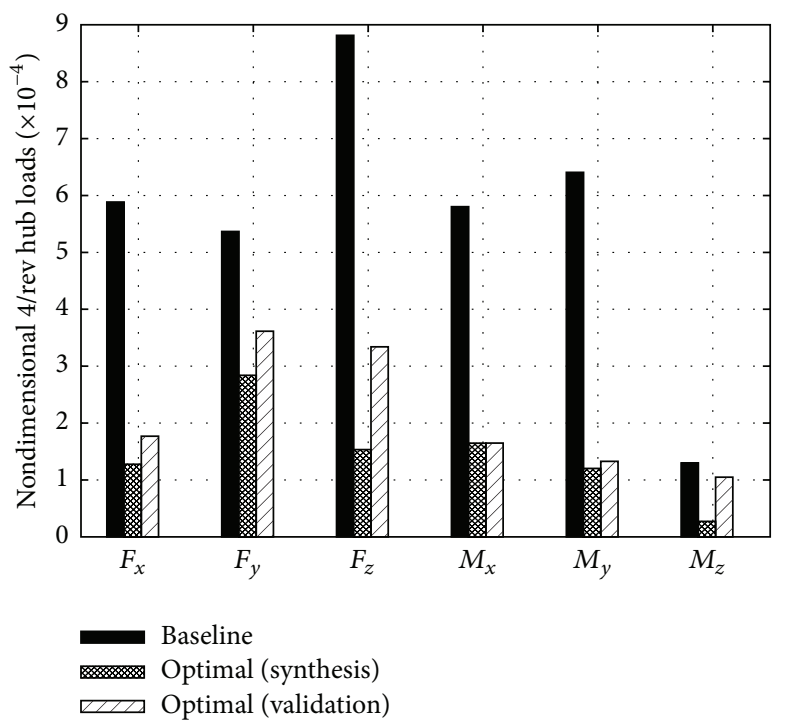

(a) Vibratory $4 /$ rev hub loads

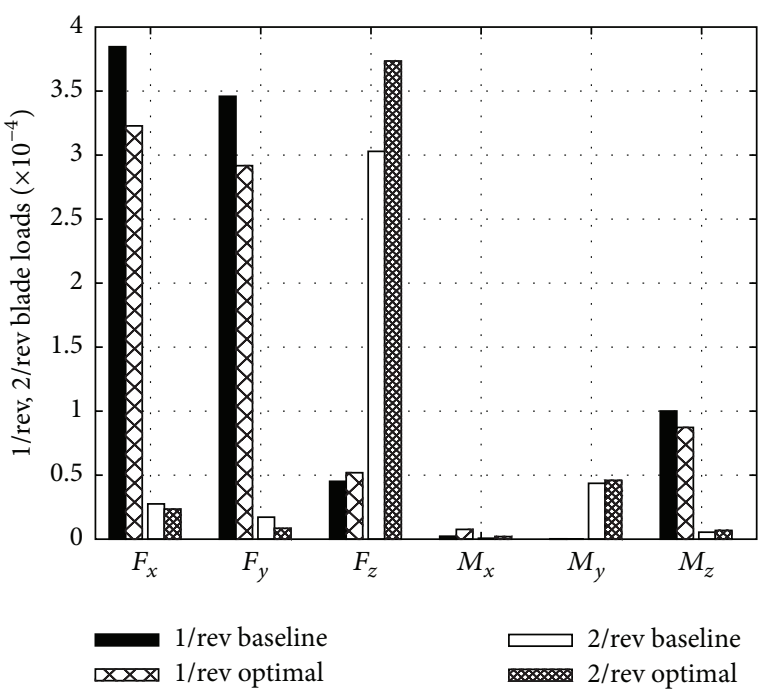

(b) Vibratory loads at blade root (validation)

FIgURE 2: Vibratory loads from single-point, surrogate wake inflow optimization; $\mu=0.3$.

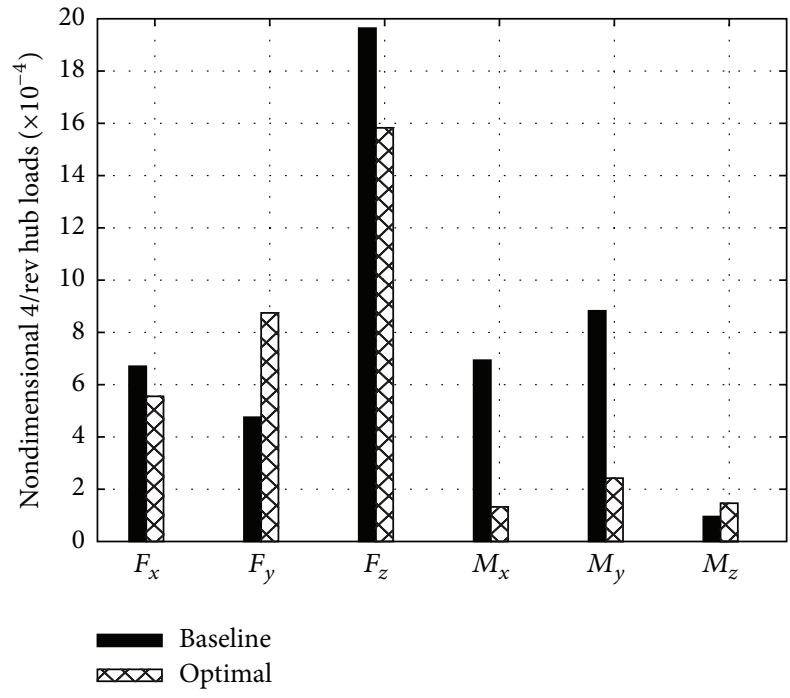

FIgURE 3: Optimized versus baseline rotor $4 / \mathrm{rev}$ hub loads at offdesign condition; $\mu=0.15$.

$\mu=0.3$, the critical eigenvalues are not appreciably affected by the blade redesign, and thus a stable behavior of the rotor is maintained. With regard to the vibratory loads, although the objective function is reduced by about $32 \%$, Figure 3 shows that the vibratory lateral shear force and torque moment are considerably increased with respect to those at the baseline configuration. In addition, it is worth noting that at $\mu=0.15$ the vibratory hub loads are higher than those at the $\mu=$ 0.3 design flight condition. The reason for this is explained by Figures 4(a) and 4(b), which depict the time histories of the blade lift spanwise distribution for the baseline rotor in the design and off-design flight conditions, respectively.
Indeed, these figures show a more irregular distribution (in space and time) of the airloads in the off-design flight condition because of the occurrence of strong blade-wake interaction effects, as revealed in Figures 5(a) and 5(b), which depict an isometric view of the computed wake geometry at the two flight conditions. Figure 5(b) clearly shows that during the low-speed flight $(\mu=0.15)$, the wake remains close to the rotor disk, thus inducing severe blade-wake impingement, at both the advancing and retreating sides of the rotor. The strong blade-wake interactions inducing higher vibratory loads at lower advance ratio may be captured by free-wake aerodynamic simulation and would remain hidden if simplified wake inflows were used (like, e.g., those based on prescribed wake shape or semianalytic ones) [39, 40]. Note also that neither advancing-side blade tip transonic effects nor retreating-side dynamic-stall/reverse flow effects are considered here, although these might significantly affect the vibratory loads in high-speed flights.

The observation that the low-speed flight condition $(\mu=$ 0.15 ) is more severe in terms of vibratory hub loads than the design flight condition, combined with the fact that the offdesign behavior of the optimal blade configuration has been proven to be unsatisfactory, suggests to apply a multipoint optimization approach in order to take into account several flight conditions in the optimization process, so as to broaden the range of the flight envelope where the optimal blade design might be effective.

3.2. Multipoint Optimization. For the multipoint optimization, the objective function has been defined as a combination of the vibratory hub loads arising at $\mu=0.15$ and $\mu=0.3$. The attempt is to develop a blade design process taking into account aerodynamic effects that characterize both high-speed and low-speed flight conditions. Specifically, the following combination of the scalar norm of the $4 / \mathrm{rev}$ 


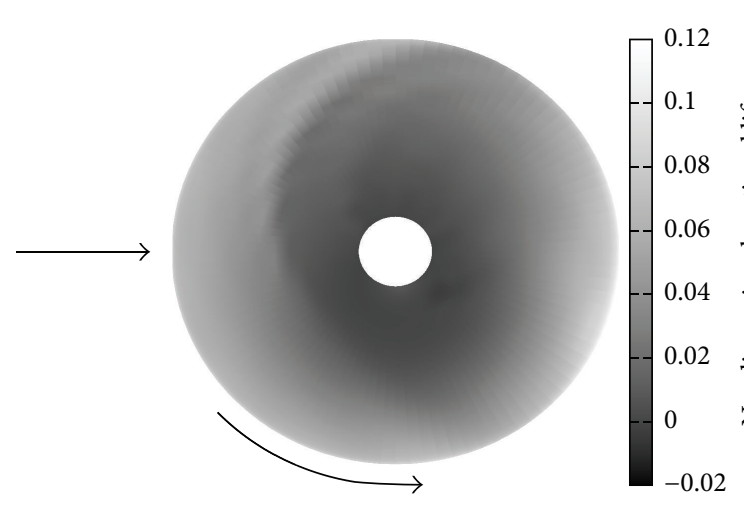

(a) Design flight condition; $\mu=0.3$

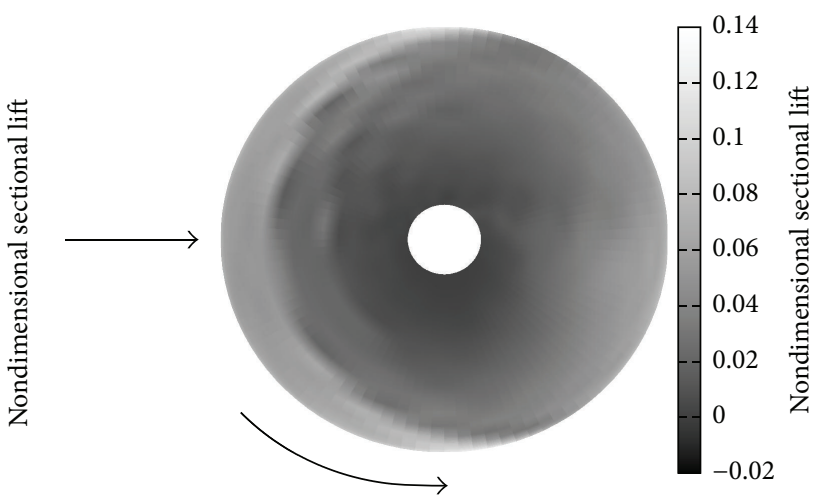

(b) Off-design flight condition; $\mu=0.15$

FIgURE 4: Rotor disk distribution of blade sectional lift.

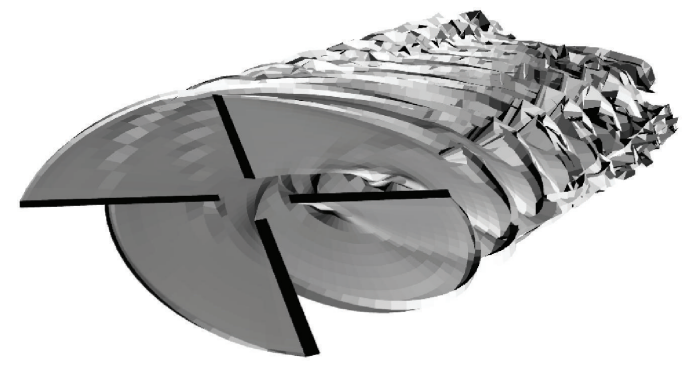

(a) Design flight condition; $\mu=0.3$

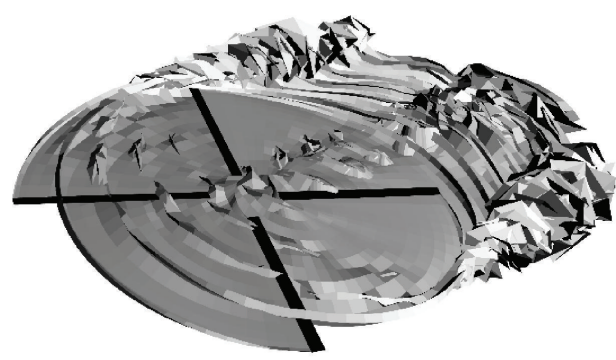

(b) Off-design flight condition; $\mu=0.15$

FIGURE 5: View of BEM free-wake geometry.

harmonics of hub forces and moments at the two flight conditions has been considered:

$$
\begin{aligned}
\widehat{J}= & \sum_{i=1}^{2}\left[\left(F_{x}^{2}+F_{y}^{2}+F_{z}^{2}\right)^{1 / 2}\right]_{i} \\
& +\left[\left(M_{x}^{2}+M_{y}^{2}+M_{z}^{2}\right)^{1 / 2}\right]_{i} .
\end{aligned}
$$

As in the single-point optimization process, the design variables are the blade mass per unit length, the bending and torsional stiffnesses, and the sweep and anhedral angles.

The result of the optimization process has been a reduction of the objective function, $\widehat{J}$, of about $64 \%$, with the optimal design variables given in the last column of Table 1. These show a reduction of bending stiffnesses with respect to the baseline values, whereas torsional stiffness and blade mass per unit length are increased. Furthermore, a rearward tip sweep angle of about $18 \mathrm{deg}$ and an upward tip anhedral angle of about 4 deg have been identified.

The corresponding $4 / \mathrm{rev}$ vibratory hub loads and $1 / \mathrm{rev}$ and $2 /$ rev blade root rotating loads are presented in Figures 6(a), 6(b), 7(a), and 7(b) for the two design flight conditions. Significant reductions of vibratory loads are evident at both design conditions, although small spillover on the in-plane shear force, $F_{y}$, and on the torque moment, $M_{z}$, is present at $\mu=0.15$ (see Figure $7(\mathrm{a})$ ). However, with these being the lowest loads, the overall quality of the results may be considered very good. In addition, these figures present the validation of the identified optimal blade against the highfidelity aerodynamic model. Indeed, vibratory loads from the surrogate inflow model used in the optimal process are quite similar to those from the more accurate BEM solution model, with the only exception of the out-of-plane shear force at $\mu=0.15$ in Figure 7(a), which is thoroughly overestimated by the solver in the optimizer (anyway, the effect of the changes of the design variables on this load seems to be well captured). The multipoint optimization has produced vibratory loads alleviation quite uniformly distributed between the two design conditions, although these alleviations, for $\mu=0.3$, are lower than those obtained by the single-point optimization procedure.

For the $1 /$ rev and $2 /$ rev rotating blade root loads, conclusions in line with those made in the case of single-point optimization can be drawn. Indeed, Figures 6(b) and 7(b) show that, although not considered in the objective function, also these loads are reduced, with the only exception of the $2 /$ rev normal shear force that is subject to some amplification both at $\mu=0.3$ and at $\mu=0.15$. Again, the increase is such that it can be considered as an acceptable minor drawback of the optimal blade configuration.

Then, the robustness of the optimal rotor configuration has been assessed by application to off-design flight 


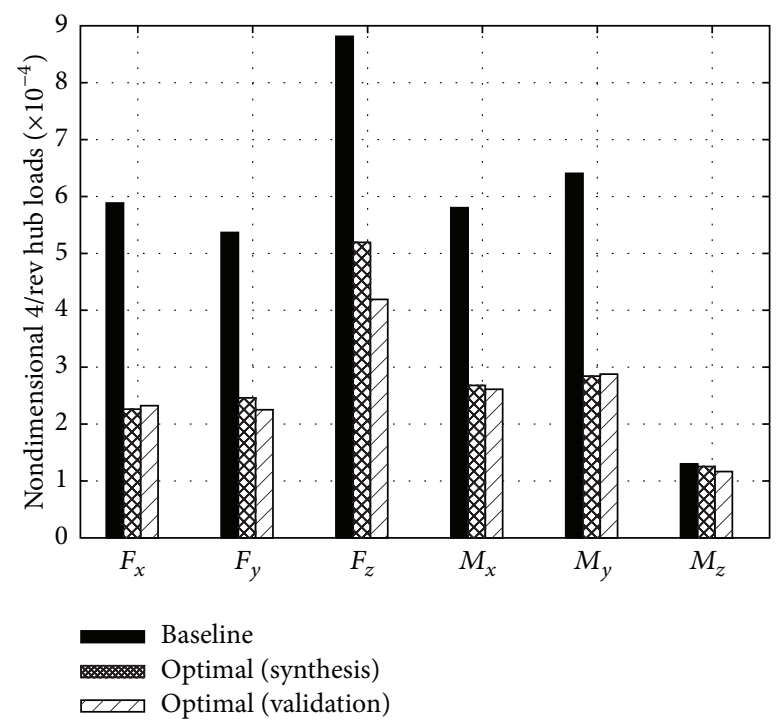

(a) Vibratory $4 /$ rev hub loads

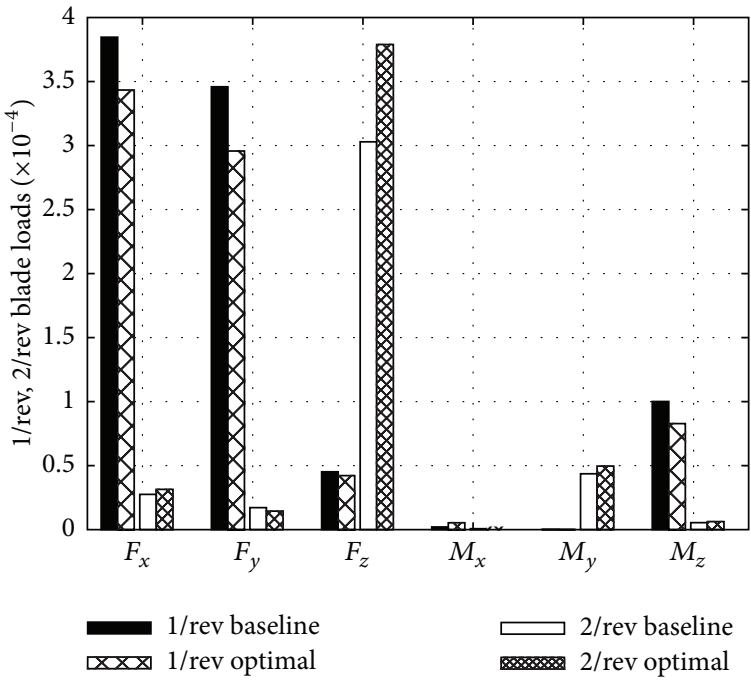

(b) Vibratory loads at blade root (validation)

FIGURE 6: Vibratory loads from multipoint, surrogate wake inflow optimization; $\mu=0.3$.

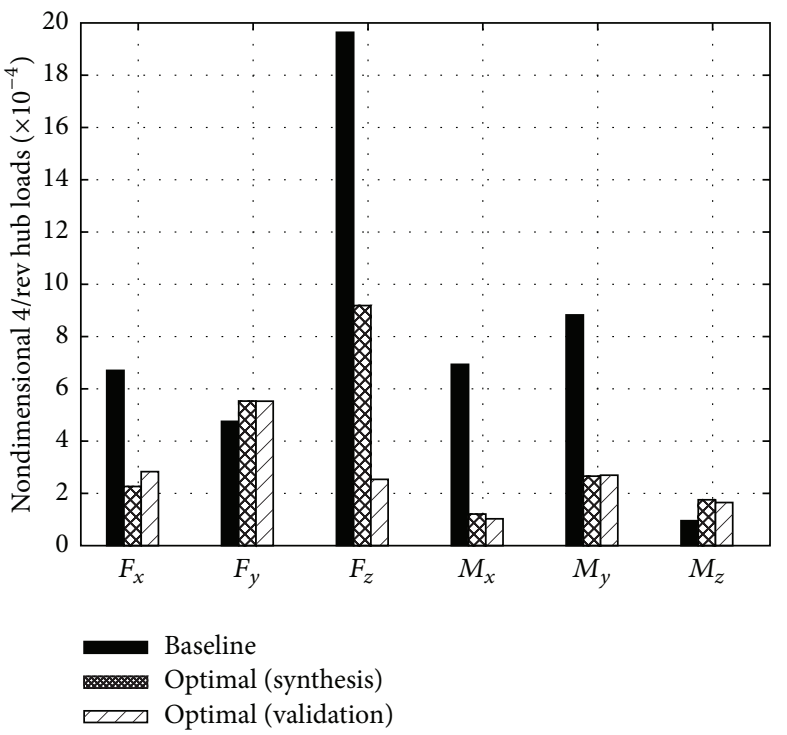

(a) Vibratory $4 /$ rev hub loads

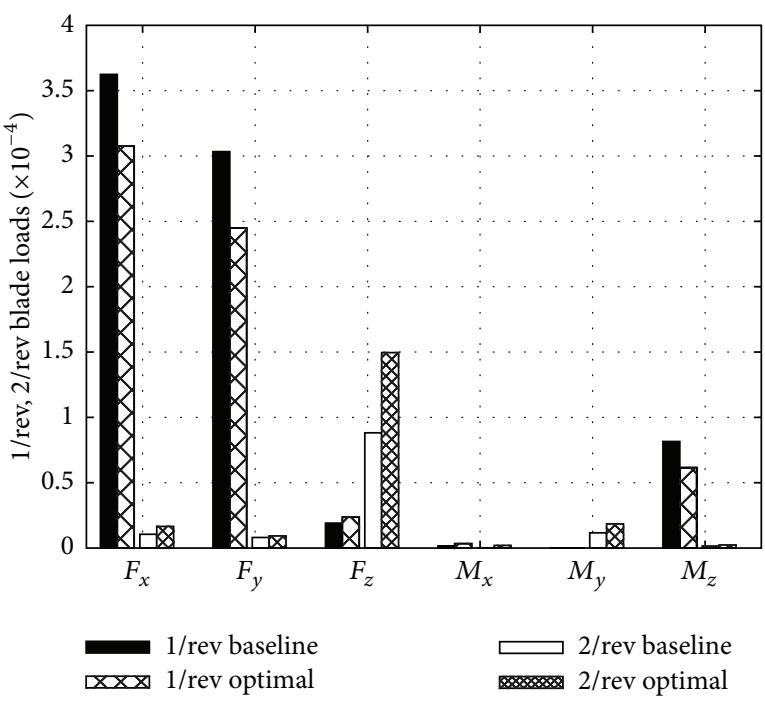

(b) Vibratory loads at blade root (validation)

FigURE 7: Vibratory loads from multipoint, surrogate wake inflow optimization; $\mu=0.15$.

conditions at advancing ratios $\mu=0.1,0.2$, and 0.25 . A stable aeroelastic response has been observed in two of these three flight conditions, with a slightly unstable eigenvalue appearing at $\mu=0.10$. However, this is not a critical issue, in that just the inclusion of a realistic structural damping in the analysis (not considered here) would have avoided the onset of such a weak instability (more generally, slight instabilities might be simply overcome by including structural dampers).

Concerning the assessment of the off-design vibratory loads generated by the optimal rotor, a hub loads magnitude index is introduced as the sum of the scalar norm of $4 / \mathrm{rev} \mathrm{hub}$ forces and moments. The values of this index computed by the high-fidelity aerodynamic model at design and off-design flight conditions are depicted in Figure 8(a). These show that, although never increased with respect to those related to the baseline blade, very small reductions are obtained at $\mu=0.10$ and $\mu=0.25$. This is essentially due to spillover effects which typically appear at off-design applications and that, here, are of particular strength at $\mu=0.10$ and $\mu=$ 0.25 . As an example, Figure 8(b) compares the optimal rotor $4 /$ rev vibratory hub loads with those from the baseline rotor, evaluated at $\mu=0.25$. Indeed, significant increases of inplane, $F_{y}$, and out-of-plane, $F_{z}$, shear forces as well as of torque moment, $M_{z}$, are observed in contrast to the reduced corresponding hub loads magnitude index in Figure 8(a). 


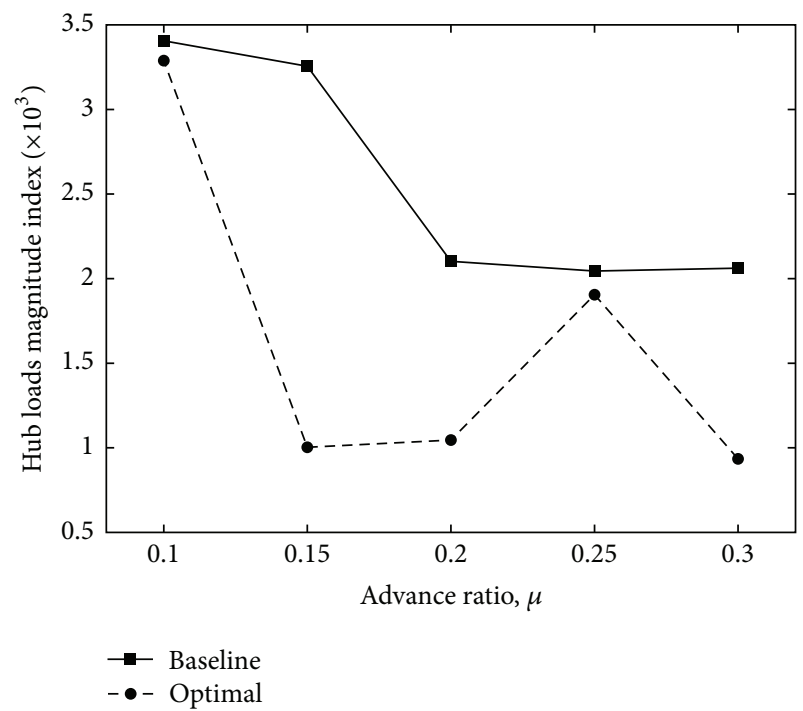

(a) Hub loads magnitude index

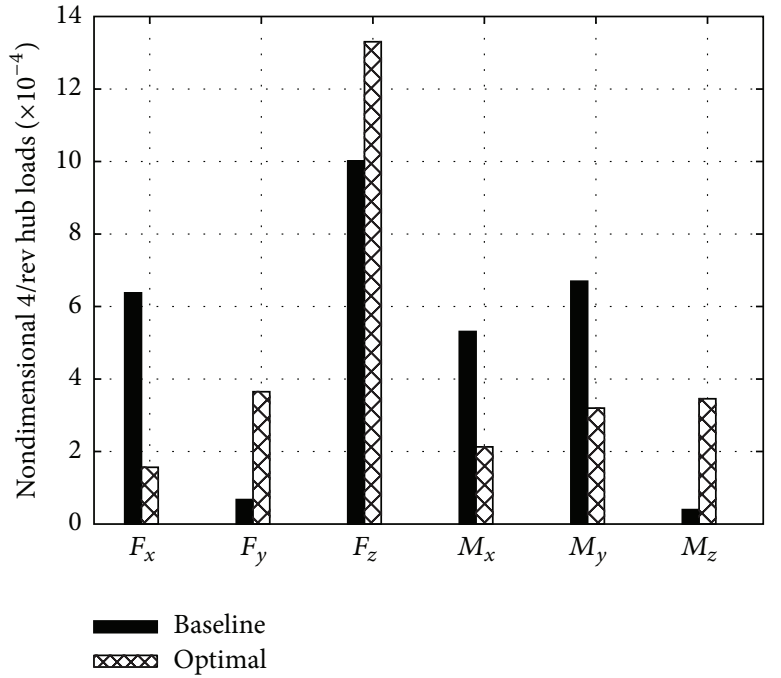

(b) Vibratory $4 /$ rev hub loads at $\mu=0.25$

FIGURE 8: Multipoint optimization: off-design conditions.

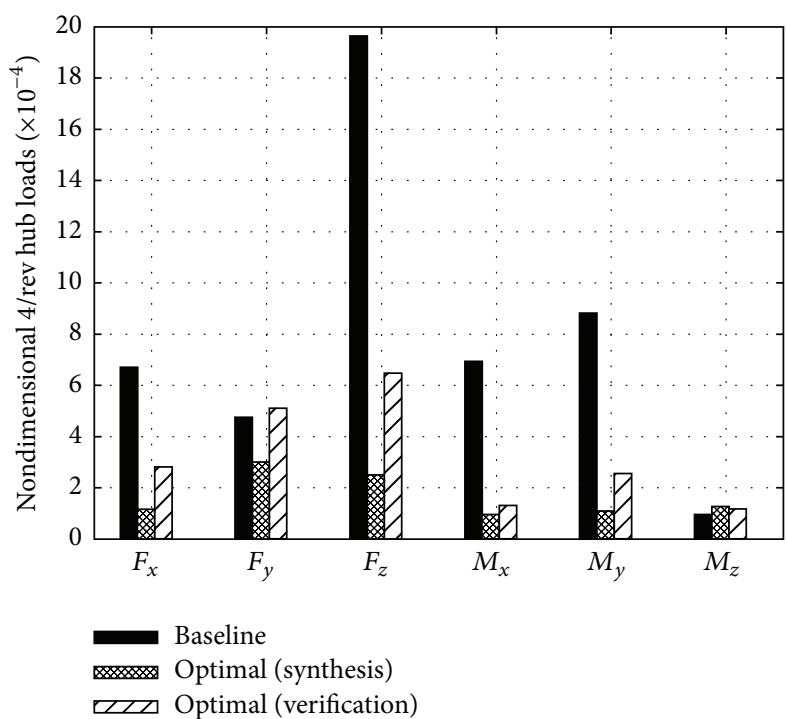

(a) Advance ratio $\mu=0.15$

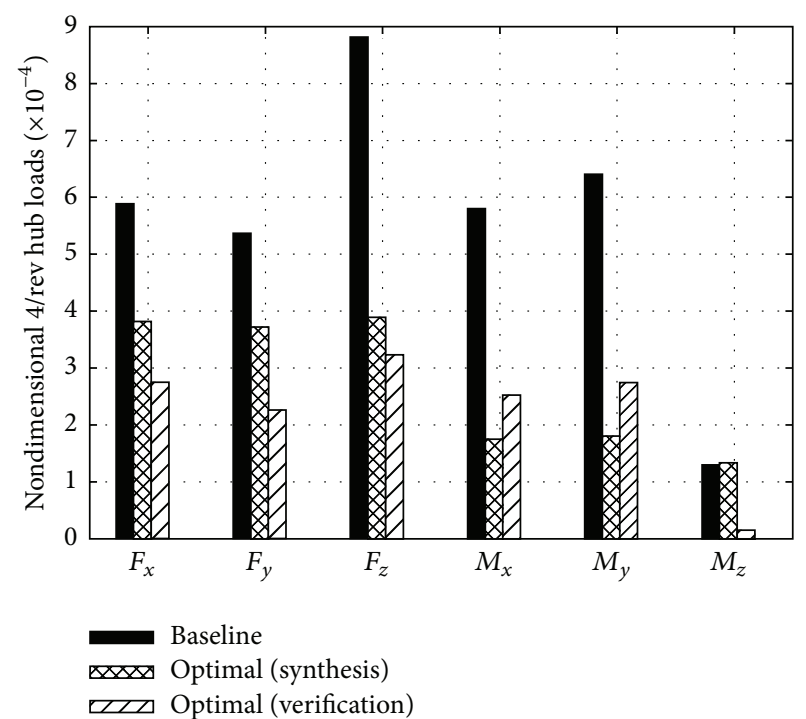

(b) Advance ratio $\mu=0.30$

FIGURE 9: Vibratory loads from multipoint, TPS surrogate wake inflow optimization.

3.3. Effects of Surrogate Models on Optimal Design. Here, the sensitivity of the multipoint optimization results to the surrogate wake inflow model is assessed. In particular, two additional techniques are investigated: thin-plate splines (TPS) and multilayer feed-forward neural networks (NN).

First, for both advance ratios considered in the multipoint optimization, the vibratory loads reduction obtained through a thin-plate spline surrogate model is presented in Figures 9(a) and 9(b). The optimal process has led to a reduction of the objective function, $\widehat{J}$, of about $62 \%$ with the design variables listed in the third column of Table 2, labeled as TPS surrogate.
The optimal configuration is similar to that obtained by using the linear wake inflow model, with a reduction of the bending stiffnesses and an increase in torsional stiffness and blade mass. Also in this case, the vibratory loads are quite similar to those estimated by the high-fidelity BEM solver, with the exception of the out-of-plane shear force at $\mu=0.15$ and the torque moment, $M_{z}$, at $\mu=0.30$.

Then, the results obtained by using a NN surrogate model are presented in Figures 10(a) and 10(b) in terms of vibratory hub loads. Also in this case, a reduction of the objective function of about $60 \%$ is achieved, with the optimal configuration given in the fourth column of Table 2. Again, the optimal 


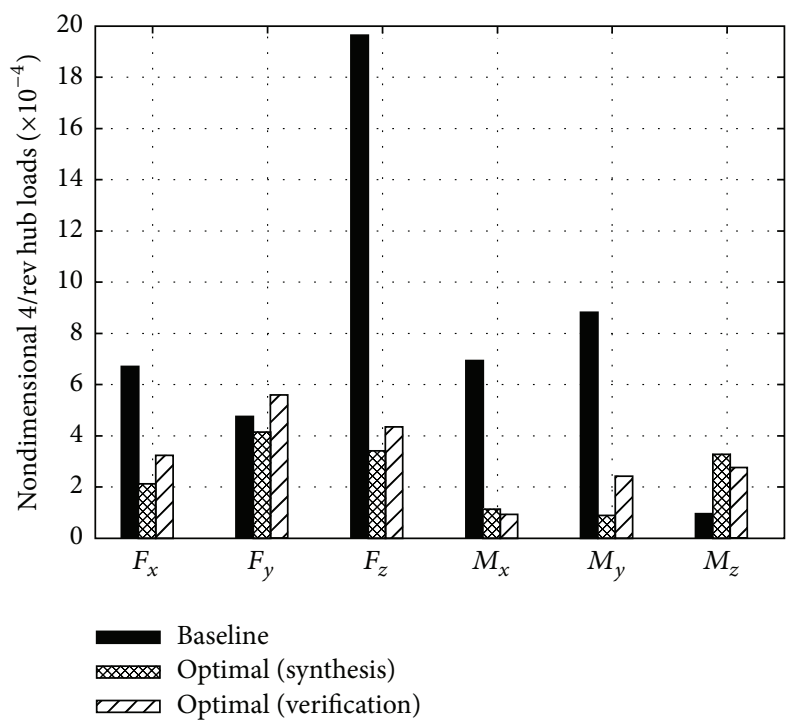

(a) Advance ratio $\mu=0.15$

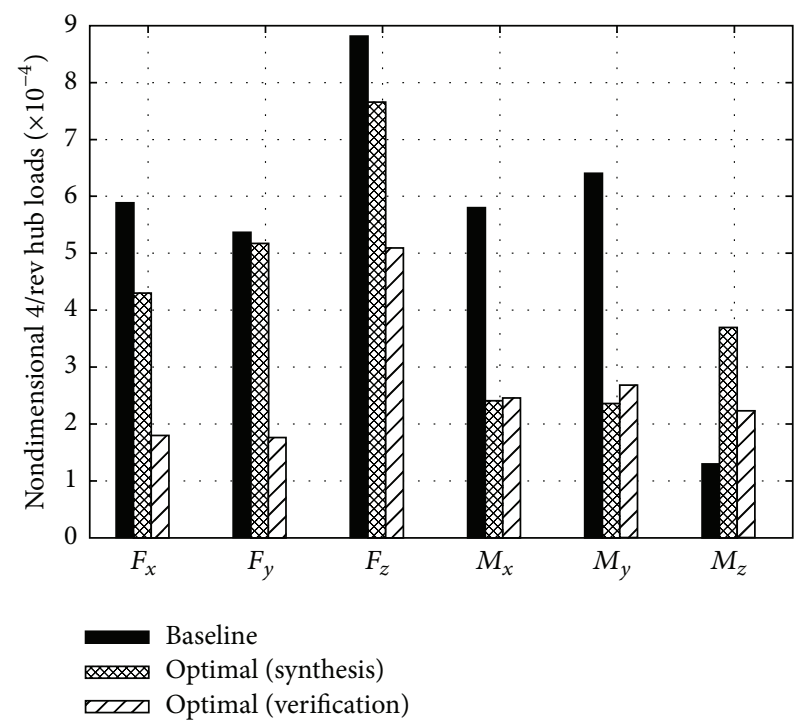

(b) Advance ratio $\mu=0.30$

FIGURE 10: Vibratory loads from multipoint, NN surrogate wake inflow optimization.

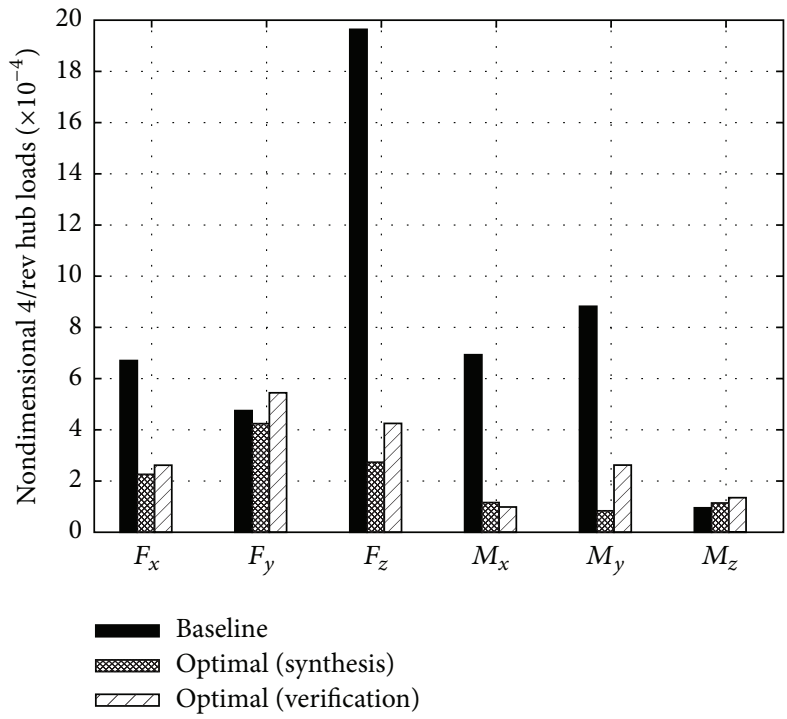

(a) Advance ratio $\mu=0.15$

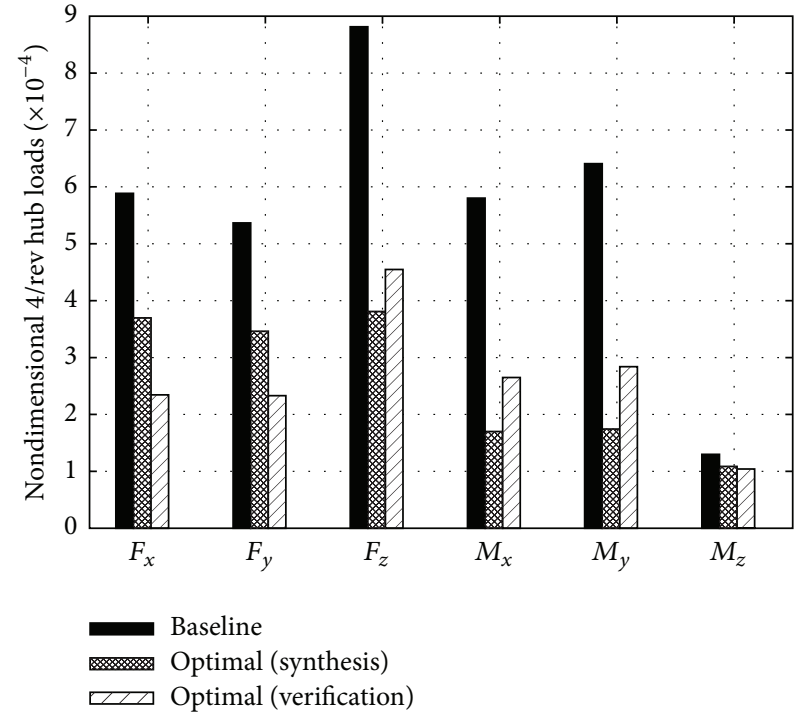

(b) Advance ratio $\mu=0.30$

FIGURE 11: Vibratory loads from multipoint, LIN-NN surrogate wake inflow optimization.

blade properties are similar to those related with the linear surrogate optimal configuration, except for the sweep angle which, in this case, is about $20 \mathrm{deg}$. The verification with the high-fidelity BEM solver has shown a satisfactory level of accuracy in the evaluation of the vibratory hub loads during the synthesis phase, with the better correlation achieved in the slower flight condition. However, some differences are present in the shear forces evaluation at $\mu=0.30$, with loads overestimated in the synthesis phase.

These results indicate that the linear surrogate model is accurate enough for the wake inflow interpolation at $\mu=$ 0.30 , whereas the neural networks interpolation is required at $\mu=0.15$. This is due to the fact that at higher advance ratios the wake is far from the rotor disk, and the wake inflow seems to be weakly influenced by the geometrical design variables, while at lower advance ratios the wake remains nearby the rotor disk with the rotor operating in a more complex aerodynamic field, thus producing stronger variations in the wake inflow when the geometrical design variables change. These observations suggest to perform a new multipoint optimization procedure, combining the two different approaches: the linear interpolation at $\mu=0.30$ and the neural networks at $\mu=0.15$.

The reduction of the objective function $\widehat{J}$ obtained is of about $62 \%$ with the design variables listed in Table 2 , column LIN-NN. Also in this case, they seem to remain similar to the 
TABLE 2: Blade design variables, multipoint optimization.

\begin{tabular}{lccccc}
\hline & Baseline & $\begin{array}{c}\text { LIN } \\
\text { surrogate }\end{array}$ & $\begin{array}{c}\text { TPS } \\
\text { surrogate }\end{array}$ & $\begin{array}{c}\text { NN } \\
\text { surrogate }\end{array}$ & $\begin{array}{c}\text { LIN-NN } \\
\text { surrogate }\end{array}$ \\
\hline$E I_{\eta} / m_{0} \Omega^{2} R^{4}$ & 0.01060 & 0.00898 & 0.00856 & 0.00910 & 0.00857 \\
$E I_{\zeta} / m_{0} \Omega^{2} R^{4}$ & 0.03010 & 0.02570 & 0.02940 & 0.02557 & 0.02664 \\
$G J / m_{0} \Omega^{2} R^{4}$ & 0.00147 & 0.00188 & 0.00187 & 0.00180 & 0.00188 \\
$m / m_{0}$ & 1.0 & 1.033 & 1.164 & 1.101 & 1.051 \\
$\Lambda_{A}[\mathrm{deg}]$ & 0.0 & -4.1 & -3.9 & -4.0 & -4.2 \\
$\Lambda_{S}[\mathrm{deg}]$ & 0.0 & 18.2 & 18.7 & 20.1 & 17.7 \\
\hline
\end{tabular}

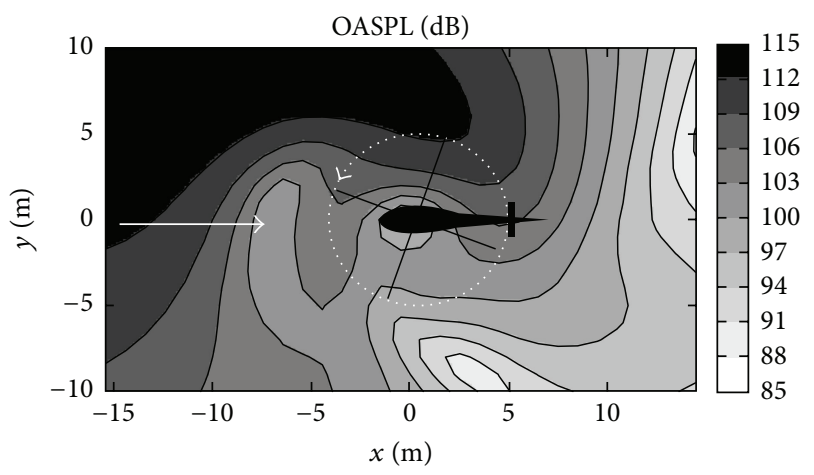

FIGURE 12: OASPL contour plot at $\mu=0.3$, baseline configuration.

other cases except, again, for the sweep angle which seems to be the variables mainly affected by the new analysis. The performances of this combined optimization, in terms of vibratory hub loads, are depicted in Figures 11(a) and 11(b): the comparison of the loads predicted through the surrogate models with those from the high-fidelity solver reveals that the accuracy of the combined surrogate model is satisfactory, although of quality similar to the results of completely LIN analysis. It is worth reminding that, in all analyses discussed, the satisfaction of the stability constraints is confirmed in the high-fidelity verification.

3.4. Assessment of the Emitted Noise. Noting that design strategies leading to reduced vibratory hub loads often result in more acoustically annoying rotors, the comparison between baseline and optimal rotor configurations is completed by the assessment of the emitted noise. To this aim, the acoustic field predicted for the optimal rotor considering the linear surrogate inflow model is considered as the representative one. The results are shown on a horizontal plane located $5.5 \mathrm{~m}$ below the rotor hub in terms of the Overall Sound Pressure Level (OASPL) that is a parameter typically considered to measure the acoustic disturbance.

Firstly, the flight condition at $\mu=0.3$ is examined. Figure 12 shows the OASPL contour plot related to the baseline rotor, whereas Figures 13(a) and 13(b) show OASPL contour plots concerning the optimal blade configurations identified through single-point and multipoint optimization procedures, respectively. The sound radiated by both optimal rotor configurations shows higher OASPL peaks (the one from the single-point optimization, in particular), along with a more pronounced directional propagation pattern. Then, the additional flight condition $(\mu=0.15)$ considered in the multipoint optimization procedure is examined. Figures 14(a) and 14(b) depict the OASPL contour plots from baseline and the optimal rotor, respectively. In this case, uniformly higher noise levels are produced by the optimal rotor throughout the entire domain considered, with slight modification of the noise radiation pattern.

These results confirm the more acoustically annoying nature of low-vibration rotors, although it is worth highlighting that in high-speed flight the observed impact may be considered low.

\section{Concluding Remarks}

Optimization procedures based on genetic, binary-based, single-point and multipoint algorithms have been developed and successfully applied for the design of rotor blades generating reduced vibratory hub loads. Aeroelastic stability constraints have been considered during the search of minimum objective function, with structural/mechanical properties, blade tip sweep angles, and anhedral angles considered as design variables. A computational tool developed by the authors for arbitrarily shaped elastic axis blades has been used to predict the aeroelastic behavior of the configurations examined in the optimization processes. Accuracy and computational efficiency has been guaranteed by application of several surrogate wake inflow models. The effects of these models on the optimal results have been investigated. The following main outcomes have been derived from the numerical investigation presented: (i) both single-point and twopoint optimizations successfully identify rotor blades suited for vibratory hub loads alleviation at the flight(s) condition(s) considered in the objective function (design conditions); (ii) increasing the number of flight conditions considered in the objective function does not reduce drastically the level of alleviation attained at each flight condition; (iii) the surrogate wake inflow models introduced are suited to be applied within the optimization process; their accuracy depends on the specific configuration examined; (iv) when operating at off-design conditions, the performance of the identified blade gets worse and spillover effect may occur (i.e., the alleviation of each vibratory load component is not guaranteed), the latter depending on the sensitivity of aerodynamic phenomena to changes of the flight configuration; (v) the aeroelastic stability of the optimal rotor is guaranteed at the design flight condition but is substantially maintained also at offdesign flights, including the critical hovering condition; (vi) the optimal design variables seem to be slightly affected by the surrogate inflow model applied, with the sweep angle being the variable most affected by it; and (vii) a minor correlation with the validation solver is shown when an analytical, poorly accurate, wake inflow model is used in the optimization procedure, the anhedral angle being the most sensitive parameter (it has opposite sign with respect to those predicted by other wake inflow models). 


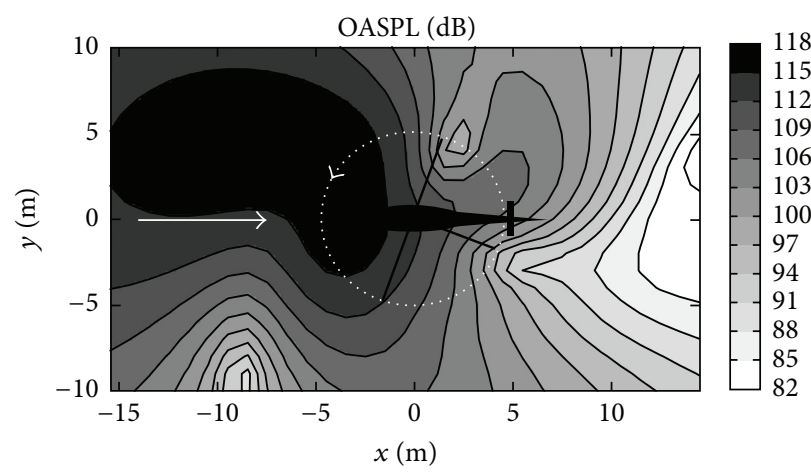

(a) Single-point optimization

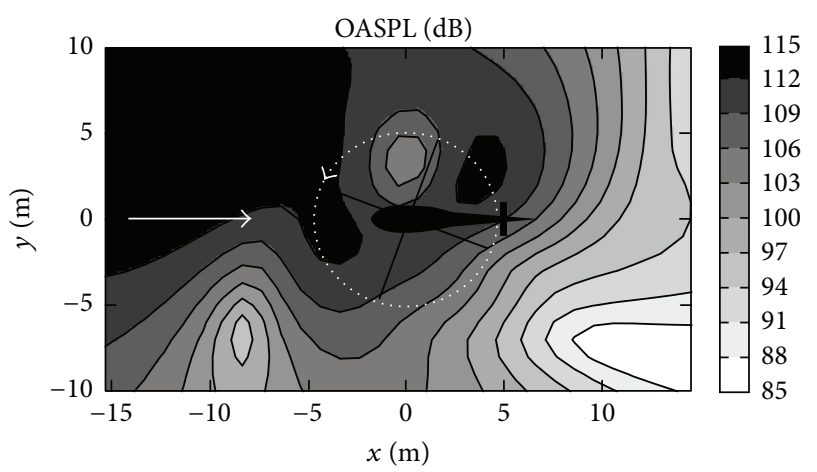

(b) Multipoint optimization

FIGURE 13: OASPL contour plot at $\mu=0.3$, optimal configurations.

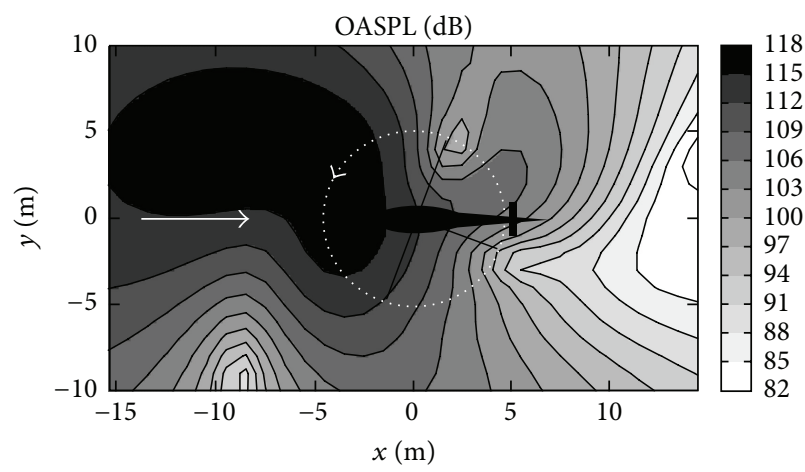

(a) Baseline

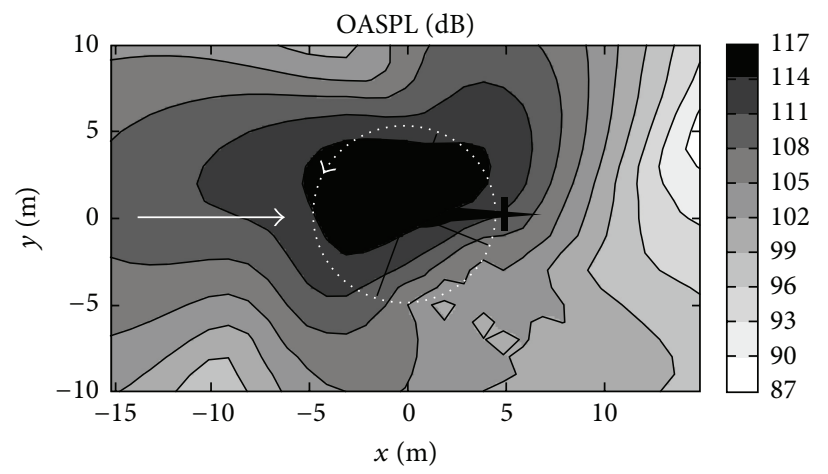

(b) Multipoint optimization

FIGURE 14: OASPL contour plot at $\mu=0.15$ from baseline and optimal configurations.

Since even the two-point optimization has been unable to guarantee significant vibration alleviation in off-design conditions, it is expected that a reduction uniformly distributed throughout the flight envelope might be achieved by either including in the objective function several flight conditions or introducing active controls to reduce vibrations in offdesign flights. The first strategy is easier to be applied in that it does not require the implementation of additional devices, but it has the drawback of yielding lower alleviation. The second one is more complex, but better performance is possibly achieved. Finally, the acoustic assessment has shown that the optimal blade configuration yields higher noise emissions, thus confirming the more acoustically annoying nature of low vibratory rotors. At high speed the increase is not relevant, while the directivity of noise pattern is significantly altered by blade redesign. Conversely, at low speed the directivity is not significantly modified, but the acoustic peaks are subject to higher increase. These results suggest the inclusion of an acoustic annoyance measure in the objective function, using multiobjective optimization techniques, so as to define a tool capable of identifying blades combining low-vibration levels with low acoustic emissions.

\section{Appendix}

\section{A. Aeroelastic Blade Model}

In the following, an outline of the mathematical formulation implemented to predict the aeroelastic behavior of rotor blades within the optimal design process is presented. First, a detailed description of the structural modeling for arbitrarily curved blades is given, followed by a brief outline of the formulation applied for the prediction of sectional loads. A concluding section presents the spectral approach applied for the spatial integration of the differential aeroelastic model.

A.1. Displacement Variables and Coordinate Systems. Several coordinate systems are introduced to derive the equations of motion of the blade. The main ones, illustrated in Figure 15, are the following:

(i) A global blade orthogonal system of unit vectors $\left(\vec{i}_{1}\right.$, $\vec{i}_{2}, \vec{i}_{3}$ ) which is centered at the rotor hub and rotates with the blade, with $\vec{i}_{1}$ tangent to the elastic axis at the root section; vectors $\vec{i}_{2}$ and $\vec{i}_{3}$ identify the principal axes of the blade root section; note that the orientation 


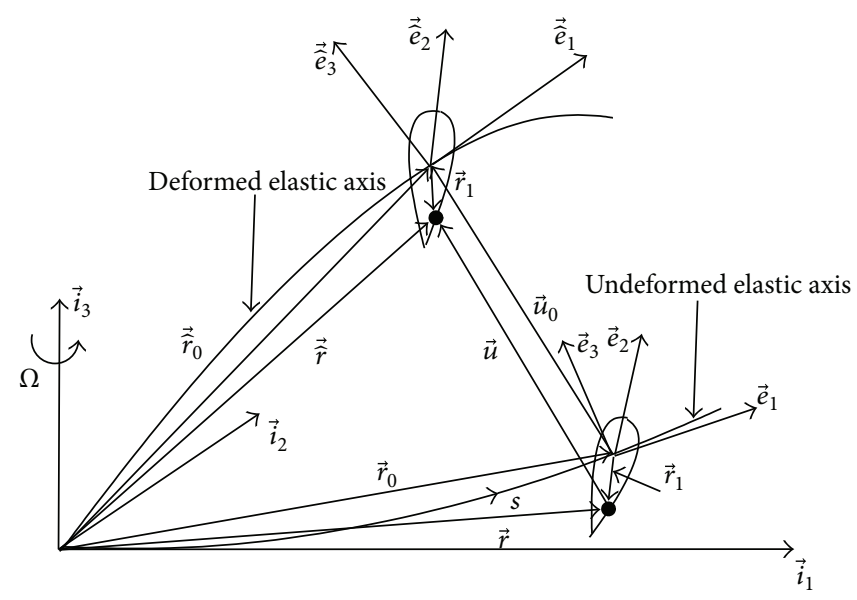

FIGURE 15: Sketch of beam representation.

of $\vec{i}_{2}$ and $\vec{i}_{3}$ depends on both collective and cyclic blade pitch commands.

(ii) Local, rotating orthogonal systems of unit vectors $\left(\vec{e}_{1}, \vec{e}_{2}, \vec{e}_{3}\right)$, with $\vec{e}_{1}$ aligned to the undeformed blade elastic axis and $\vec{e}_{2}, \vec{e}_{3}$ aligned with the blade section principal axes; note that the orientation of $\vec{e}_{2}$ and $\vec{e}_{3}$ depends also on blade pretwist.

(iii) Local, rotating orthogonal systems related to the blade deformed configuration having base unit vectors $\left(\overrightarrow{\hat{e}}_{1}, \overrightarrow{\widehat{e}}_{2}, \overrightarrow{\hat{e}}_{3}\right)$, with $\overrightarrow{\widehat{e}}_{1}$ tangent to the deformed elastic axis and $\overrightarrow{\hat{e}}_{2}, \vec{e}_{3}$ aligned with the principal axes of the elastically twisted blade sections (this assumption means that the sections of the deformed beam remain orthogonal to the deformed elastic axis and hence a shear undeformable beam model is considered).

Deformations are described in terms of displacements of the elastic axis and rotation of beam sections. The displacements, $u, v, w$, are defined in the local frame fixed with the undeformed blade, respectively, along the directions identified by $\vec{e}_{1}, \vec{e}_{2}$, and $\vec{e}_{3}$; the blade twist, $\phi$, is defined as the rotation of blade sections about $\vec{e}_{1}$-direction (i.e., about the deformed elastic axis).

In the development of the blade dynamics formulation, the definition of transformation matrices relating the frames of reference defined above is also convenient.

Global to Undeformed Blade References. The unit base vectors of the global rotating frame are related to those of the localundeformed blade frames through the following expression:

$$
\left\{\vec{e}_{i}\right\}=\mathbf{A}(s)\left\{\vec{i}_{i}\right\}
$$

where, for $\vec{e}_{i}=\vec{e}_{1}, \vec{e}_{2}, \vec{e}_{3}$, and $s$ denoting the curvilinear coordinate defined along the undeformed elastic axis, $\mathbf{A}(s)$ is the transformation matrix yielding the local frame unit vectors in terms of superposition of the global-frame base (i.e., it collects the components of each $\vec{e}_{i}$ in the global frame).
Blade-Undeformed to Blade Deformed References. Localundeformed blade and deformed blade references are related by the following expression:

$$
\left\{\overrightarrow{\hat{e}}_{i}\right\}=\mathbf{T}(s)\left\{\vec{e}_{i}\right\}
$$

where $\mathbf{T}$ is the transformation matrix depending on the deformation of the blade. The matrix $\mathbf{T}$ is obtained as a sequence of rotations about $\vec{e}_{3}$, (rotated) $\vec{e}_{2}$, and (rotated) $\vec{e}_{1}$; its expression in terms of $u, v, w, \phi$ may be derived from that given in [41].

Global to Blade Deformed References. From the transformations mentioned above it is possible to determine the relation between global and deformed blade references as

$$
\left\{\overrightarrow{\widetilde{e}}_{i}\right\}=\Lambda(s)\left\{\vec{i}_{i}\right\}
$$

where $\Lambda=$ TA.

A.2. Equilibrium Relations. Considering a deformed beam element of length $\mathrm{d} s$, the equilibrium of forces and moments acting on it yields

$$
\begin{array}{r}
\frac{\mathrm{d} \vec{v}}{\mathrm{~d} s}+\vec{p}=0, \\
\frac{\mathrm{d} \vec{m}}{\mathrm{~d} s}+\overrightarrow{\widehat{e}}_{1} \times \vec{v}+\vec{q}=0,
\end{array}
$$

where $\vec{v}$ and $\vec{m}$ are the internal structural forces and moments at the elastic axis, while $\vec{p}$ and $\vec{q}$ are the external distributed forces and moments.

In order to integrate the above differential equilibrium equations, it is convenient to write them in terms of forces and moments components, $\mathbf{v}_{l}, \mathbf{m}_{l}, \mathbf{p}_{l}$, and $\mathbf{q}_{l}$, in the local bladeundeformed frames. Observing that, for $\mathbf{v}_{g}, \mathbf{m}_{g}, \mathbf{p}_{g}$, and $\mathbf{q}_{g}$ denoting forces and moments components in the global frame of reference, one has $\mathbf{v}_{g}=\mathbf{A}^{T} \mathbf{v}_{l}, \mathbf{m}_{g}=\mathbf{A}^{T} \mathbf{m}_{l}, \mathbf{p}_{g}=$ 
$\mathbf{A}^{T} \mathbf{p}_{l}$, and $\mathbf{q}_{g}=\mathbf{A}^{T} \mathbf{q}_{l}$, the equilibrium equations projected onto the frame $\left\{\vec{i}_{i}\right\}$ yield

$$
\begin{aligned}
\frac{\mathrm{d}}{\mathrm{d} s}\left(\mathbf{A}^{T} \mathbf{v}_{l}\right)+\mathbf{A}^{T} \mathbf{p}_{l} & =0, \\
\frac{\mathrm{d}}{\mathrm{d} s}\left(\mathbf{A}^{T} \mathbf{m}_{l}\right)-\mathbf{A}^{T} \mathbf{H}_{l} \mathbf{v}_{l}+\mathbf{A}^{T} \mathbf{q}_{l} & =0,
\end{aligned}
$$

where $\mathbf{H}_{l}$ is the matrix of the components in the localundeformed frame of the axial tensor associated to vector $\overrightarrow{\hat{e}}_{1}$.

Next, integration of (A.5) yields the following distribution of the internal shear loads:

$$
\mathbf{v}_{l}(s)=\mathbf{A} \int_{s}^{R} \mathbf{A}^{T} \mathbf{p}_{l} \mathrm{~d} \widehat{s},
$$

while the integration of (A.6) yields the following distribution of the internal moments:

$$
\begin{aligned}
& \mathbf{m}_{l}(s) \\
& =\mathbf{A}\left\{\int_{s}^{R} \mathbf{A}^{T} \mathbf{q}_{l} \mathrm{~d} \widehat{s}-\int_{s}^{R}\left(\mathbf{A}^{T} \mathbf{H}_{l} \mathbf{A} \int_{\widehat{s}}^{R} \mathbf{A}^{T} \mathbf{p}_{l} \mathrm{~d} \widehat{s}\right) \mathrm{d} \widehat{s}\right\},
\end{aligned}
$$

where $R$ denotes the length of the undeformed elastic axis (under assumption of negligible second-order terms related to local slope).

Equations (A.7) and (A.8) are the general solutions for the internal shear loads and moments arising in a beam, from which the equations governing the blade elastic displacement may be derived, once strain-displacement and loaddisplacement relations are identified (see next subsections). Note that, because of shear undeformable assumption, the equations governing the blade motion variables, $u, v, w$ and $\phi$, are derived from the first scalar equation in (A.7) and the three scalar equations in (A.8) (the second and third scalar equations in (A.7) are used to determine the shear load components lying in the plane of beam sections).

A.3. Strain-Displacement Relations. In order to express the internal loads in terms of the (shear undeformable) beam deformation variables, $u, v, w$, and $\phi$, the strain tensor has to be derived from position vectors describing undeformed and deformed beam. The position vector, $\vec{r}$, of a point of the undeformed beam of coordinates $(s, \eta, \zeta)$, with $\eta$ and $\zeta$ denoting the coordinates along the principal axes of the sections, is given by $\vec{r}(s, \eta, \zeta)=\vec{r}_{0}+\eta \vec{e}_{2}+\zeta \vec{e}_{3}$, while after deformation, under the assumption of negligible warping, its position is identified by the following vector: $\vec{r}(s, \eta, \zeta)=$ $\vec{r}_{0}+u \vec{e}_{1}+v \vec{e}_{2}+w \vec{e}_{3}+\eta \overrightarrow{\widehat{e}}_{2}+\zeta \overrightarrow{\hat{e}}_{3}$, where $\vec{r}_{0}$ denotes the position of the points along the undeformed elastic axis. From the above equations the strain tensor, $\vec{E}$, is derived from

$$
\vec{E}(s, \eta, \zeta)=\frac{1}{2}\left(\frac{\partial \vec{r}}{\partial \xi^{i}} \cdot \frac{\partial \vec{r}}{\partial \xi^{j}}-\frac{\partial \vec{r}}{\partial \xi^{i}} \cdot \frac{\partial \vec{r}}{\partial \xi^{j}}\right) \frac{\partial \vec{r}}{\partial \xi^{i}} \otimes \frac{\partial \vec{r}}{\partial \xi^{j}},
$$

where $\left(\xi^{1}, \xi^{2}, \xi^{3}\right) \equiv(s, \eta, \zeta)$, observing that the local coordinate systems considered are orthogonal.
A.4. Internal Load-Displacement Relations. From the stressstrain relations given by the theory of elasticity combined with the strain tensor expression, it is possible to derive the stress-displacement relations and in turn those between internal structural loads and displacement variables, after integration over the beam sections. Under the assumptions of rigid beam sections and shear undeformable beam, the following four loads are given in terms of the four variables describing the beam motion (the evaluation of the remaining two loads comes from balancing of the external loads):

$$
\begin{aligned}
V_{\widehat{x}} & =\vec{v} \cdot \overrightarrow{\widehat{e}}_{1}=\int_{A} \sigma_{x x} \mathrm{~d} \eta \mathrm{d} \zeta \\
& =E A\left(\widetilde{u}^{\prime}+\frac{\widetilde{v}^{\prime 2}}{2}+\frac{\widetilde{w}^{\prime 2}}{2}+k_{A}^{2} k_{1}^{2}\right), \\
M_{\widehat{\eta}} & =\vec{m} \cdot \vec{e}_{2}=-\int_{A} \sigma_{x x} \zeta \mathrm{d} \eta \mathrm{d} \zeta=E I_{\eta} k_{2}, \\
M_{\widetilde{\zeta}} & =\vec{m} \cdot \vec{e}_{3}=\int_{A} \sigma_{x x} \eta \mathrm{d} \eta \mathrm{d} \zeta=E I_{\zeta} k_{3}, \\
M_{\widehat{x}} & =\vec{m} \cdot \vec{e}_{1}=\int_{A}\left(\sigma_{x \eta} \eta-\sigma_{x \zeta} \zeta\right) \mathrm{d} \eta \mathrm{d} \zeta \\
& =G J k_{1}+V_{\widehat{x}} k_{A}^{2} k_{1},
\end{aligned}
$$

where $A$ is the blade cross-section area, $E$ is the Young modulus, and $G$ is the shear modulus, while

$$
\begin{aligned}
I_{\eta} & =\int_{A} \zeta^{2} \mathrm{~d} \eta \mathrm{d} \zeta, \\
J & =\int_{A}\left(\eta^{2}+\zeta^{2}\right) \mathrm{d} \eta \mathrm{d} \zeta, \\
I_{\zeta} & =\int_{A} \eta^{2} \mathrm{~d} \eta \mathrm{d} \zeta, \\
k_{A}^{2} & =\frac{1}{A} \int_{A}\left(\eta^{2}+\zeta^{2}\right) \mathrm{d} \eta \mathrm{d} \zeta .
\end{aligned}
$$

In addition, $k_{1}, k_{2}, k_{3}$ are such that, for $\vec{k}=k_{1} \overrightarrow{\hat{e}}_{1}+k_{2} \overrightarrow{\hat{e}}_{2}+$ $k_{3} \vec{e}_{3}$, the skew-symmetric matrix $\mathbf{K}=(\mathrm{d} \mathbf{T} / \mathrm{d} s) \mathbf{T}^{T}$ is the matrix of the components in the local-deformed frame of the axial tensor associated with vector $\vec{k}$ (specifically, $k_{2}$ and $k_{3}$ are the bending curvatures of the deformed elastic axis, whereas $k_{1}$ is the twist of the blade sections after deformation). Finally, following a second-order geometric description, the extensional deformation of the elastic axis is given by $\epsilon_{x x}=\widetilde{u}^{\prime}+\widetilde{v}^{\prime 2} / 2+\widetilde{w}^{\prime 2} / 2$, where $\widetilde{u}^{\prime}, \widetilde{v}^{\prime}$, and $\widetilde{w}^{\prime}$ are the components of $\mathrm{d} \widetilde{\mathbf{u}} / \mathrm{d} s=\mathrm{d} \mathbf{u} / \mathrm{d} s-\mathbf{K}_{0} \mathbf{u}$, for $\mathbf{u}=\left\{\begin{array}{lll}u & v & w\end{array}\right\}^{T}$ and $\mathbf{K}_{0}=(\mathrm{d} \mathbf{A} / \mathrm{d} s) \mathbf{A}^{T}$.

A.5. Inertial Loads. Blade rotation and unsteady deformations make inertial loads arise. These contribute to the equilibrium equations as external distributed loads and, combined with the internal loads, yield the equations governing 
blade structural dynamics. The acceleration of a generic point of a rotating blade is given by

$$
\vec{a}=\vec{a}_{r}+\vec{a}_{H}+\vec{\Omega} \times \vec{\Omega} \times \overrightarrow{\widehat{r}}+2 \vec{\Omega} \times \vec{v},
$$

where $\vec{a}_{r}$ is the acceleration of the point with respect to a frame rigidly connected to the undeformed blade. It is derived from the rigid motion of the beam cross sections expressed in terms of displacements of the elastic axis and rotations about it. In addition, $\vec{a}_{H}$ is the rotor hub acceleration, and $\vec{v}$ is the velocity of the examined point with respect to the rotating frame fixed with the undeformed blade, while $\vec{\Omega}$ is the blade angular velocity. Then, the resulting inertial distributed loads appearing in (A.4) are expressed as

$$
\begin{aligned}
& \vec{p}=-\int_{A} \rho \vec{a} \mathrm{~d} \eta \mathrm{d} \zeta, \\
& \vec{q}=-\int_{A} \rho \vec{r}_{s} \times \vec{a} \mathrm{~d} \eta \mathrm{d} \zeta
\end{aligned}
$$

where $\rho$ is the material density and $\vec{r}_{s}=\vec{r}-\left(\vec{r}_{0}+u \vec{e}_{1}+v \vec{e}_{2}+\right.$ $\left.w \vec{e}_{3}\right)$.

A.6. Equations for Deformation Variables. In order to apply the structural formulation outlined above, it is necessary to derive the relationship between the blade displacement unknowns, $u, v, w$, and $\phi$, appearing in the definition of the inertial loads and the deformation variables, $\epsilon_{x x}, \widehat{k}_{1}, \widehat{k}_{2}$, and $\widehat{k}_{3}$, appearing in the expressions of the internal loads.

To this purpose, the vector of the cross-section rotations about the axes of the local-deformed frame of reference is introduced, observing that the derivative of its global-frame components with respect to the curvilinear abscissa, $s$, gives the components, in the same frame, of vector $\vec{k}$ of Section A.4. Reminding the shear undeformable beam assumption, this yields

$$
\mathbf{A} \frac{\partial\left(\mathbf{A}^{T} \boldsymbol{\theta}\right)}{\partial s}=\mathbf{k}
$$

and hence

$$
\boldsymbol{\theta}(s)=\mathbf{A} \int_{0}^{s} \mathbf{A}^{T} \mathbf{k} \mathrm{d} s,
$$

where $\boldsymbol{\theta}$ denotes the vector of the local-undeformed frame components of the cross-section rotations, while $\mathbf{k}$ denotes the components of $\vec{k}$ in the same frame. Note that the first component of $\boldsymbol{\theta}$ corresponds to the elastic torsion, $\phi$, whereas the second and third ones are related to the displacement components $u, v$, and $w$ through the following second-order nonlinear kinematic relation:

$$
\mathbf{A} \frac{\partial \mathbf{A}^{T} \mathbf{u}}{\partial s}=\left\{\begin{array}{c}
\epsilon_{x x}-f_{\mathrm{nl}}(u, v, w) \\
\theta_{3} \\
-\theta_{2}
\end{array}\right\}
$$

where the nonlinear term, $f_{\mathrm{nl}}$, takes into account the extension of the elastic axis due to bending.
Thus, the deformation variables $\epsilon_{x x}, \widehat{k}_{1}, \widehat{k}_{2}$, and $\widehat{k}_{3}$ appearing in the expression of the internal elastic loads are related to $u, v, w$, and $\phi$ through combination of (A.15) and (A.16).

Note that, from the combination of the above kinematic relations with the internal loads, the inertial loads, the first scalar equation in (A.5), and the three scalar equations in (A.6), it is possible to derive a set of four integrodifferential equations in the deformation unknowns, $u, v, w, \phi$, governing the structural dynamics of a blade with arbitrarily curved elastic axis.

A.7. Aerodynamic Loads. As already mentioned in the main body text, the aerodynamic loads are derived from a quasisteady approximation of the Greenberg theory [28] for airfoils. Aerodynamic three-dimensional effects are taken into account by including wake inflow (either through an analytical model or by an aerodynamic solution tool). Thus, section force, $T$, orthogonal to the chord, and $S$, parallel to the chord are given by

$$
\begin{aligned}
& T=\frac{\varrho C_{l_{\alpha}} c}{2}\left[-U_{P} U_{T}+\frac{c}{2} \omega U_{T}-\frac{c}{4} \dot{U}_{P}+\left(\frac{c}{4}\right)^{2} \dot{\omega}\right], \\
& S=\frac{\varrho C_{l_{\alpha}} c}{2}\left[U_{P}^{2}-\frac{c}{2} \omega U_{P}-\frac{C_{d_{0}}}{C_{l_{\alpha}}} U_{T}^{2}\right],
\end{aligned}
$$

while the section pitching moment with respect to the quarter-chord point reads

$$
M_{\phi}=-\frac{\varrho C_{l_{\alpha}} c^{3}}{32}\left(\omega U_{T}-\dot{U}_{P}+\frac{3 c}{8} \dot{\omega}\right) .
$$

In the above equations, $U_{P}$ and $U_{T}$ are, respectively, the quarter-chord velocity components normal and parallel to the chord after deformation; $\omega$ is the out-of-section component of the angular velocity of the blade section; $c$ denotes the chord length; $\varrho$ is the air density; $C_{l_{\alpha}}$ is the lift curve slope coefficient, while $C_{d_{0}}$ is the drag coefficient.

For including these equations within an aeroelastic model, $U_{P}, U_{T}$, and $\omega$ are expressed in terms of $u, v, w$, and $\phi$ and the aerodynamic forces $T$ and $S$ are projected onto the local blade-undeformed frame of reference.

A.8. Spectral Solution. In the numerical solver developed, the blade elastic axis is considered inextensible. In this case the axial degree of freedom, $u$, is derived as a consequence of beam bending (akin to the approach followed in [29]). The corresponding set of governing equations is that in (A.8), with unknown variables $v, w$, and $\phi$.

To explain the procedure adopted for the numerical solution of these equations, it is convenient to recast them formally as

$$
\mathbf{m}_{l}=\mathbf{m}_{\mathrm{in}}+\mathbf{m}_{\mathrm{aer}},
$$

where $\mathbf{m}_{\text {in }}$ and $\mathbf{m}_{\text {aer }}$ denote the inertial and aerodynamic contributions to the RHS of (A.8), respectively. The spatial integration of (A.19) is obtained through a spectral approach. 
The first step consists of the following description of the undeformed-axis frame components of the curvatures:

$$
\begin{aligned}
& k_{1}(s, t)=\sum_{n=1}^{N_{1}} \alpha_{n}^{\phi}(t) \psi_{n}^{\phi^{\prime}}(s), \\
& k_{2}(s, t)=\sum_{n=1}^{N_{2}} \alpha_{n}^{w}(t) \psi_{n}^{w^{\prime \prime}}(s), \\
& k_{3}(s, t)=\sum_{n=1}^{N_{3}} \alpha_{n}^{v}(t) \psi_{n}^{v^{\prime \prime}}(s),
\end{aligned}
$$

where $\psi_{n}^{\phi}, \psi_{n}^{v}$, and $\psi_{n}^{w}$ may be conveniently chosen as the torsion and bending natural modes of vibration of a nonrotating beam [29]. Note that, in (A.20), the first-order derivative of the torsion shape function, $\psi_{n}^{\phi}$, is used in that related to the elastic twist of the blade, $k_{1}$, whereas in (A.21) and (A.22) the second-order derivatives of the bending shape functions, $\psi_{n}^{v}$ and $\psi_{n}^{w}$, have been introduced in that related to the bending curvatures, $k_{2}$ and $k_{3}$.

Then the equations in (A.19) are projected onto the same set of functions applied in (A.20)-(A.22):

$$
\int_{0}^{R} \mathbf{m}_{l}^{T} \boldsymbol{\Psi} \mathrm{d} s=\int_{0}^{R}\left(\mathbf{m}_{\mathrm{in}}^{T}+\mathbf{m}_{\mathrm{aer}}^{T}\right) \boldsymbol{\Psi} \mathrm{d} s
$$

where

$$
\Psi=\left\{\begin{array}{c}
\psi_{n}^{\phi^{\prime}} \\
\psi_{n}^{w^{\prime \prime}} \\
\psi_{n}^{\nu^{\prime \prime}}
\end{array}\right\} .
$$

The choice of using the first- and second-order derivatives of the shape functions as test functions is mainly motivated by the equivalence between the resulting equations and those that would be derived from application of the Rayleigh-Ritz approach. The resulting aeroelastic system consists of a set of $\left(N_{1}+N_{2}+N_{3}\right)$ nonlinear, time-dependent equations with unknowns $\alpha$, which can be applied for both aeroelastic response and stability analysis.

\section{Nomenclature}

$\begin{array}{ll}c: & \text { Blade chord } \\ C_{T}: & \begin{array}{l}\text { Rotor thrust coefficient, } T / \rho \pi \Omega^{2} R^{4}(T= \\ \text { thrust, } \rho=\text { air density })\end{array} \\ E I_{\eta}, E I_{\zeta}: & \text { Bending stiffnesses } \\ F_{x}, F_{y}, F_{z}: & \text { Hub force components } \\ G J: & \text { Torsional stiffness } \\ \widehat{J}: & \text { Objective function } \\ m: & \text { Mass distribution } \\ m_{0}: & \text { Baseline mass distribution } \\ M_{x}, M_{y}, M_{z}: & \text { Hub moment components } \\ R: & \text { Rotor radius }\end{array}$

$V: \quad$ Hub-freestream relative velocity

$\gamma$ : Lock number, $3 \rho a c R / m_{0}(a=$ airfoil lift curve slope coefficient)

$\Lambda_{A}$ : Blade tip anhedral angle (positive downwards)

$\Lambda_{S}:$ Blade tip sweep angle (positive backwards)

$\mu$ : $\quad$ Advance ratio, $V / \Omega R$

$\sigma: \quad$ Rotor solidity

$\Omega$ : Rotor blade angular speed.

\section{Competing Interests}

The authors declare that they have no competing interests.

\section{References}

[1] P. Rauch, M. Gervais, P. Cranga et al., "Blue edge: the design, development and testing of a new blade concept," in Proceedings of the 67th Annual Forum of the American Helicopter Society, pp. 542-555, Virginia Beach, Va, USA, 2011.

[2] Y. H. Yu, "Rotor blade-vortex interaction noise," Progress in Aerospace Sciences, vol. 36, no. 2, pp. 97-115, 2000.

[3] K. A. Yuan and P. P. Friedmann, "Aeroelasticity and structural optimization of composite helicopter rotor blades with swept tips," NASA CR-4665, 1995.

[4] K.-A. Yuan and P. P. Friedmann, "Structural optimization for vibratory loads reduction of composite helicopter rotor blades with advanced geometry tips," Journal of the American Helicopter Society, vol. 43, no. 3, pp. 246-256, 1998.

[5] R. Ganguli and I. Chopra, "Aeroelastic optimization of an advanced geometry helicopter rotor," Journal of the American Helicopter Society, vol. 41, no. 1, pp. 18-29, 1996.

[6] R. Ganguli and I. Chopra, "Aeroelastic optimization of a helicopter rotor with two-cell composite blades," AIAA Journal, vol. 34, no. 4, pp. 835-841, 1996.

[7] R. Ganguli and I. Chopra, "Aeroelastic optimization of a helicopter rotor to reduce vibration and dynamic stresses," Journal of Aircraft, vol. 33, no. 4, pp. 808-815, 1996.

[8] S. Murugan and R. Ganguli, "Inuence of inow models on helicopter aeroelastic optimization," Computational Fluid Dynamics Journal, vol. 16, no. 4, pp. 444-453, 2008.

[9] B. Glaz, T. Goel, L. Liu, P. P. Friedmann, and R. T. Haftka, "Multiple-surrogate approach to helicopter rotor blade vibration reduction," AIAA Journal, vol. 47, no. 1, pp. 271-282, 2009.

[10] B. Glaz, P. P. Friedmann, and L. Liu, "Helicopter vibration reduction throughout the entire flight envelope using surrogatebased optimization," Journal of the American Helicopter Society, vol. 54, no. 1, pp. 1-15, 2009.

[11] P. P. Friedmann, "Helicopter vibration reduction using structural optimization with aeroelastic/multidisciplinary constraints-a survey," Journal of Aircraft, vol. 28, no. 1, pp. 8-21, 1991.

[12] R. Celi, "Recent applications of design optimization to rotorcraft-a survey," Journal of Aircraft, vol. 36, no. 1, pp. 176189, 1999.

[13] R. Ganguli, "A survey of recent developments in rotorcraft design optimization," Journal of Aircraft, vol. 41, no. 3, pp. 493510, 2004.

[14] G. Bernardini, C. Testa, and M. Gennaretti, "Optimal design of tonal noise control inside smart-stiffened cylindrical shells," 
Journal of Vibration and Control, vol. 18, no. 8, pp. 1233-1246, 2012.

[15] D. Calcagni, G. Bernardini, and F. Salvatore, "Automated marine propeller optimal design combining hydrodynamics models and neural networks," in Proceedings of 11th International Conference on Computer Applications and Information Technology in the Maritime Industries, Liege, Belgium, January 2012.

[16] J. H. Holland, Adaptation in Nature and Artificial Systems, University of Michigan Press, Ann Arbor, Mich, USA, 1975.

[17] D. E. Goldberg, "Optimal initial population size for binarycoded genetic algorithms," TCGA Report 85001, University of Alabama, Tuscaloosa, Ala, USA, 1985.

[18] J. R. R. A. Martins and A. B. Lambe, "Multidisciplinary design optimization: a survey of architectures," AIAA Journal, vol. 51, no. 9, pp. 2049-2075, 2013.

[19] R. Celi, Aeroelasticity and structural optimization of helicopter rotor blades with swept tips [Ph.D. thesis], Mechanical, Aerospace and Nuclear Engineering Department, University of California, Los Angeles, Calif, USA, 1987.

[20] R. Celi and P. P. Friedmann, "Aeroelastic modeling of swept tip rotor blades using finite elements," Journal of the American Helicopter Society, vol. 33, no. 2, pp. 43-52, 1988.

[21] G. S. Bir and I. Chopra, "Aeromechanical stability of rotorcraft with advanced geometry blades," Mathematical and Computer Modelling, vol. 19, no. 3-4, pp. 159-191, 1994.

[22] V. A. Riziotis, S. G. Voutsinas, D. I. Manolas, E. S. Politis, and P. K. Chaviaropoulos, "Aeroelastic analysis of pre-curved rotor blades," in Proceedings of the European Wind Energy Conference and Exhibition (EWEC '10), Warsaw, Poland, April 2010.

[23] E. Piccione, G. Bernardini, M. Molica Colella, and M. Gennaretti, "Structural and aeroelastic modeling of curved rotor blades using a galerkin approach," in Proceedings of the $3 \mathrm{rd}$ Ceas Air \& Space Conference/21st AIDAA Congress, pp. 615-624, Venice, Italy, 2011.

[24] E. Piccione, G. Bernardini, M. Molica Colella, and M. Gennaretti, "A spectral formulation for structural/aeroelastic modeling of curved-axis rotor blades," Aerotecnica Missili \& Spazio, vol. 91, no. 1-2, pp. 42-52, 2012.

[25] M. Gennaretti and G. Bernardini, "Novel boundary integral formulation for blade-vortex interaction aerodynamics of helicopter rotors," AIAA Journal, vol. 45, no. 6, pp. 1169-1176, 2007.

[26] F. Farassat, "Derivation of formulations 1 and 1 A of Farassat," NASA TM-2007-214853, 2007.

[27] J. E. Ffowcs Williams and D. L. Hawkings, "Sound generated by turbulence and surfaces in arbitrary motion," Philosophical Transactions of the Royal Society A, vol. 264, no. 1151, pp. 321342, 1969.

[28] J. M. Greenberg, "Airfoil in sinusoidal motion in pulsating stream," NACA TN-1326, 1947.

[29] D. H. Hodges and R. A. Ormiston, "Stability of elastic bending and torsion of uniform cantilever rotor blades in hover with variable structural coupling," NASA TN D-8192, 1976.

[30] M. Gennaretti and G. Bernardini, "Aeroelastic response of helicopter rotors using a 3D unsteady aerodynamic solver," The Aeronautical Journal, vol. 110, no. 1114, pp. 793-801, 2006.

[31] M. Gennaretti and G. Bernardini, "Aeroacousto-elastic modeling for response analysis of helicopter rotors," in Variational Analysis and Aerospace Engineering: Mathematical Challenges for Aerospace Design, G. Buttazzo and A. Frediani, Eds., vol. 66 of Springer Optimization and Its Applications, pp. 27-50, Springer, Berlin, Germany, 2012.
[32] R. E. Smith and E. Smuda, "Adaptively resizing populations: algorithm, analysis, and first results," Complex Systems, vol. 9, no. 1, pp. 47-72, 1995.

[33] R. T. Haftka and Z. Gürdal, Elements of Structural Optimization, Kluwer Academic, Dordrecht, The Netherlands, 1992.

[34] G. Rudolph, "Evolutionary search under partially ordered fitness sets," in Proceedings of the International Symposium on Information Science Innovations in Engineering of Natural and Artificial Intelligent Systems (ISI '01), pp. 818-822, ICSC Academic Press, 2001.

[35] E. Zitzler, K. Deb, and L. Thiele, "Comparison of multiobjective evolutionary algorithms: empirical results," Evolutionary Computation, vol. 8, no. 2, pp. 173-195, 2000.

[36] D. P. Raymer, Enhancing aircraft conceptual design using multidisciplinary optimization [Ph.D. thesis], Royal Institute of Technology, Stockholm, Sweden, 2002.

[37] C. Venkatesan, P. P. Friedmann, and K.-A. Yuan, "A new sensitivity analysis for structural optimization of composite rotor blades," Mathematical and Computer Modelling, vol. 19, no. 3-4, pp. 1-25, 1994.

[38] R. Ganguli, "Optimum design of a helicopter rotor for low vibration using aeroelastic analysis and response surface methods," Journal of Sound and Vibration, vol. 258, no. 2, pp. 327-344, 2002.

[39] J. Zhang, E. C. Smith, and K. W. Wang, "Active-passive hybrid optimization of rotor blades with trailing edge flaps," Journal of the American Helicopter Society, vol. 49, no. 1, pp. 54-65, 2004.

[40] A. Datta and I. Chopra, "Validation and understanding of UH-60A vibratory loads in steady level flight," Journal of the American Helicopter Society, vol. 49, no. 3, pp. 271-287, 2004.

[41] D. H. Hodges and E. H. Dowell, "Nonlinear equations of motion for the elastic bending and torsion of twisted nonuniform rotor blades," NASA TN D-7818, 1974. 


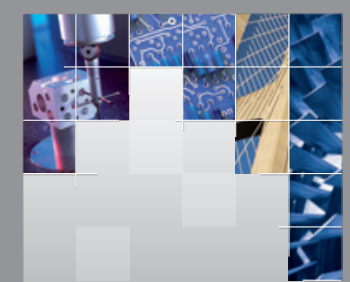

\section{Enfincering}
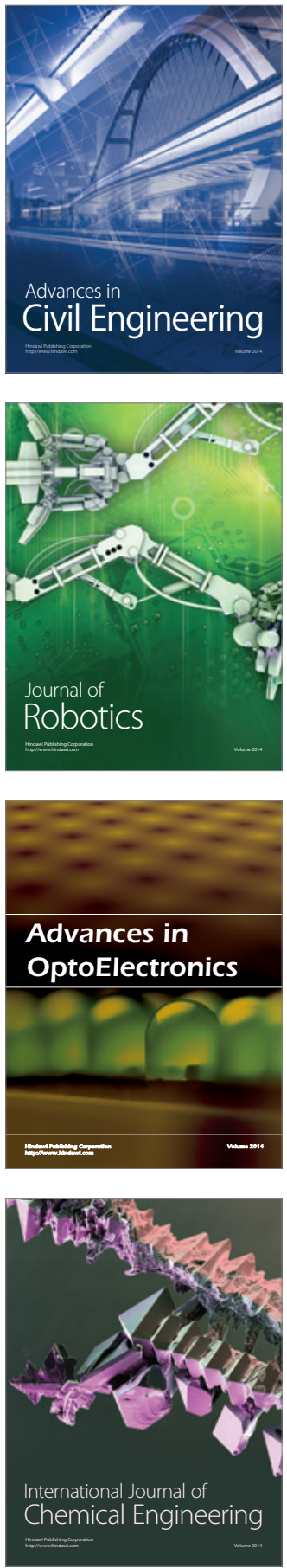

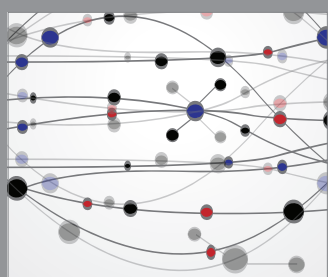

The Scientific World Journal

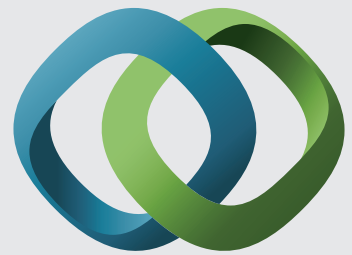

\section{Hindawi}

Submit your manuscripts at

http://www.hindawi.com
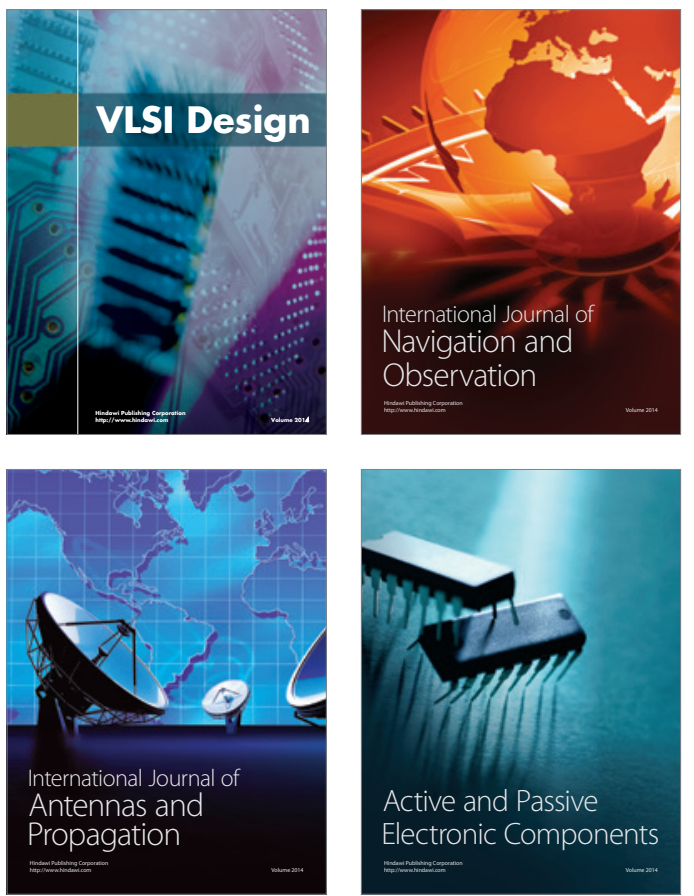
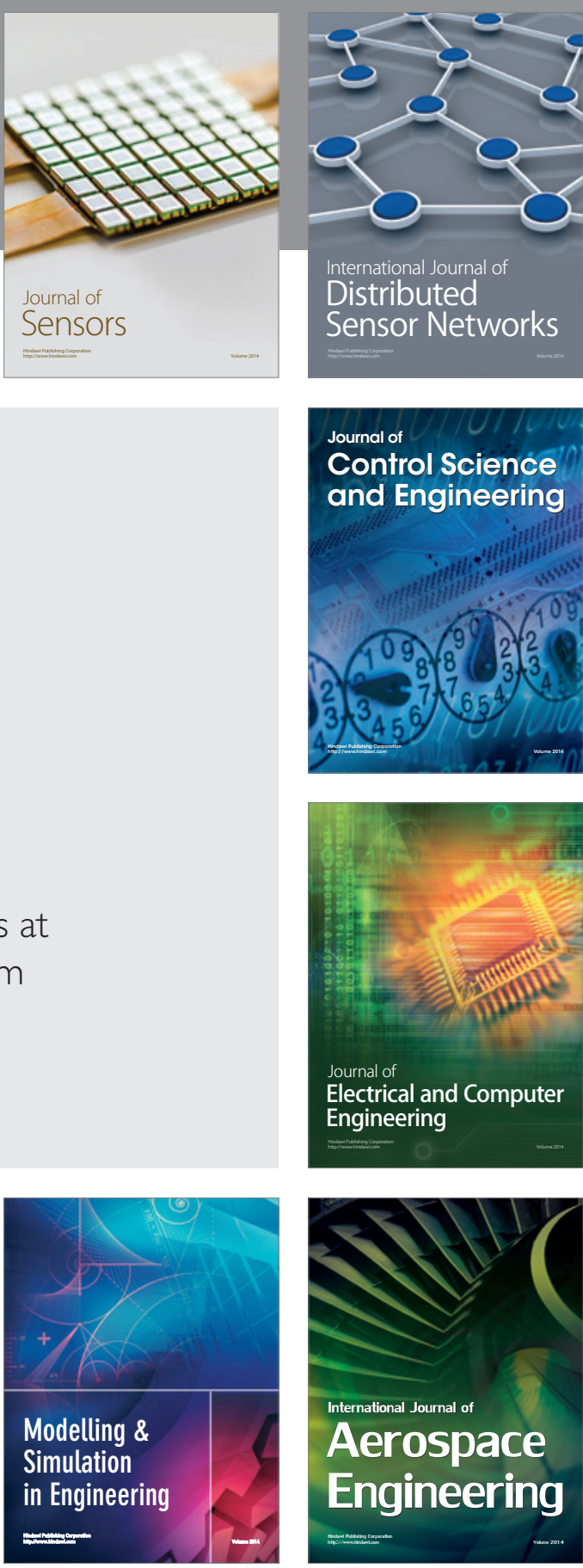

International Journal of

Distributed

Sensor Networks

Journal of

Control Science

and Engineering
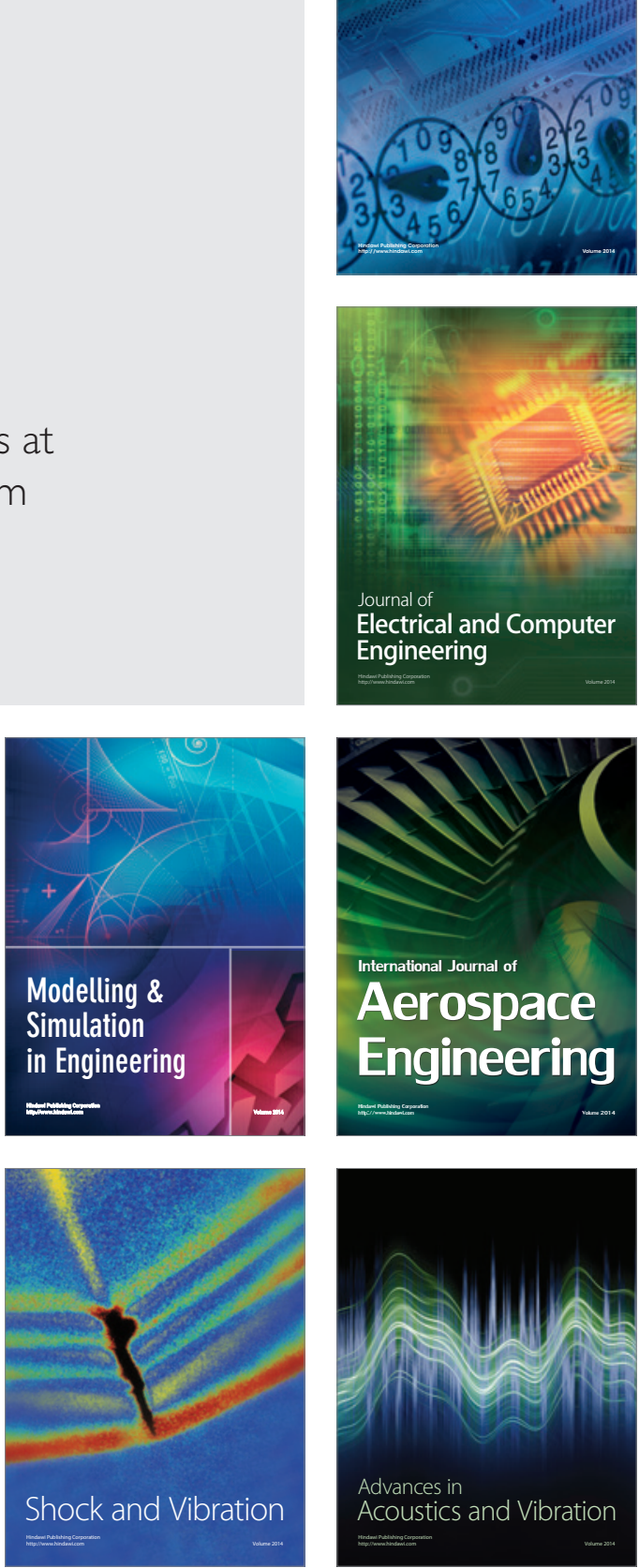